\title{
Denitrification by large NAT particles: the impact of reduced settling velocities and hints on particle characteristics
}

\author{
W. Woiwode ${ }^{1}$, J.-U. Grooß ${ }^{2}$, H. Oelhaf ${ }^{1}$, S. Molleker ${ }^{3}$, S. Borrmann ${ }^{3,4}$, A. Ebersoldt ${ }^{5}$, W. Frey ${ }^{3, *}$, T. Gulde ${ }^{1}$, \\ S. Khaykin ${ }^{6, * *}$, G. Maucher ${ }^{1}$, C. Piesch ${ }^{1}$, and J. Orphal ${ }^{1}$ \\ ${ }^{1}$ Institute for Meteorology and Climate Research, Karlsruhe Institute of Technology, Karlsruhe, Germany \\ ${ }^{2}$ Institute of Energy and Climate Research - Stratosphere (IEK-7), Forschungszentrum Jülich, Jülich, Germany \\ ${ }^{3}$ Max Planck Institute for Chemistry (MPI), Particle Chemistry Department, Mainz, Germany \\ ${ }^{4}$ Institute for Physics of the Atmosphere (IPA), University of Mainz, Mainz, Germany \\ ${ }^{5}$ Institute for Data Processing and Electronics, Karlsruhe Institute of Technology, Karlsruhe, Germany \\ ${ }^{6}$ Central Aerological Observatory, Dolgoprudny, Moscow region, Russia \\ *now at: School of Earth Sciences, The University of Melbourne, Melbourne, Victoria, Australia \\ ** now at: LATMOS, CNRS-INSU, Université de Versailles St. Quentin, Guyancourt, France
}

Correspondence to: W. Woiwode (wolfgang.woiwode@ kit.edu)

Received: 16 January 2014 - Published in Atmos. Chem. Phys. Discuss.: 6 March 2014

Revised: 1 September 2014 - Accepted: 15 September 2014 - Published: 31 October 2014

\begin{abstract}
Vertical redistribution of $\mathrm{HNO}_{3}$ through large $\mathrm{HNO}_{3}$-containing particles associated with polar stratospheric clouds (PSCs) plays an important role in the chemistry of the Arctic winter stratosphere. During the RECONCILE (Reconciliation of essential process parameters for an enhanced predictability of Arctic stratospheric ozone loss and its climate interactions) campaign, apparently very large NAT (nitric acid trihydrate) particles were observed by the airborne in situ probe FSSP-100 (Molleker et al., 2014). Our analysis shows that the FSSP-100 observations associated with the flight on 25 January 2010 cannot easily be explained assuming compact spherical NAT particles due to much too short growing time at temperatures below the existence temperature of NAT $\left(T_{\mathrm{NAT}}\right)$. State-of-the-art simulations using CLaMS (Chemical Lagrangian Model of the Stratosphere; Grooß et al., 2014) suggest considerably smaller particles. We consider the hypothesis that the simulation reproduces the NAT particle masses in a realistic way, but that real NAT particles may have larger apparent sizes compared to compact spherical particles, e.g. due to non-compact morphology or aspheric shape. Our study focuses on the consequence that such particles would have reduced settling velocities compared to compact spheres, altering the vertical redistribution of $\mathrm{HNO}_{3}$. Utilising CLaMS simulations, we investigate the impact of reduced settling velocities of NAT particles on ver-
\end{abstract}

tical $\mathrm{HNO}_{3}$ redistribution and compare the results with observations of gas-phase $\mathrm{HNO}_{3}$ by the airborne Fourier transform spectrometer MIPAS-STR associated with two RECONCILE flights. The MIPAS-STR observations confirm conditions consistent with denitrification by NAT particles for the flight on 25 January 2010 and show good agreement with the simulations within the limitations of the comparison. Best agreement is found if settling velocities between 100 and $50 \%$ relative to compact spherical particles are considered (slight preference for the $70 \%$ scenario). In contrast, relative settling velocities of $30 \%$ result in too weak vertical $\mathrm{HNO}_{3}$ redistribution. Sensitivity simulations considering temperature biases of $\pm 1 \mathrm{~K}$ and multiplying the simulated nucleation rates by factors of 0.5 and 2.0 affect the comparisons to a similar extent, but result in no effective improvement compared to the reference scenario. Our results show that an accurate knowledge of the settling velocities of NAT particles is important for quantitative simulations of vertical $\mathrm{HNO}_{3}$ redistribution. 


\section{Introduction}

Irreversible vertical redistribution of $\mathrm{HNO}_{3}$ through denitrification plays an important role in Arctic ozone depletion chemistry (Solomon, 1999, and references therein). The freezing-out of $\mathrm{HNO}_{3}$-containing particles at altitudes above ca. $18 \mathrm{~km}$ followed by sedimentation delays the deactivation of ozone-destroying substances by limiting $\mathrm{NO}_{\mathrm{x}}$ (reactive nitrogen oxide radicals) availability from $\mathrm{HNO}_{3}$ photolysis. Furthermore, the formation of liquid and solid polar stratospheric cloud (PSC) particles and the reactive surface capable of chlorine activation depend on $\mathrm{HNO}_{3}$ availability in the gas phase (Grooß et al., 2005, and references therein). Particles capable of denitrification are assumed to be composed of NAT (nitric acid trihydrate), ice-coated NAT (or vice versa) and potentially also further metastable phases composed of $\mathrm{HNO}_{3}$ and $\mathrm{H}_{2} \mathrm{O}$, with NAD (nitric acid dihydrate) being one of the most likely candidates (Hanson and Mauersberger, 1988; Worsnop et al., 1993; Peter and Grooß, 2012, and references therein). Potentially higher hydrates of $\mathrm{HNO}_{3}$ are also reported in the literature (e.g. Marti and Mauersberger, 1994; Tabazadeh and Toon, 1996), while experimental evidence is sparse. Measurements of large $\mathrm{HNO}_{3}$-containing particles with significant potential to denitrify the lower stratosphere were reported by Fahey et al. (2001).

In situ observations performed with the FSSP-100 (Forward Scattering Spectrometer Probe 100) during the Arctic RECONCILE (Reconciliation of essential process parameters for an enhanced predictability of Arctic stratospheric ozone loss and its climate interactions) field campaign in early 2010 aboard the high-altitude research aircraft M-55 Geophysica indicate potential NAT particles with unexpected large sizes and high number densities (von Hobe et al., 2013; Molleker et al., 2014). Such large NAT particles cannot easily be explained with current theory of particle growth and sedimentation assuming approximately spherical shape, compact morphology and particle mass density described in the literature, as will be shown in this work for measurements made during the flight on 25 January 2010.

The properties of micrometre-sized ice particles in the atmosphere are well known (Libbrecht, 2005, and references therein). Depending on the crystallisation conditions, plates, needles and more complex crystals are found, with many of the particles found being considerably aspheric. For aspheric ice particles, Westbrook (2008) estimated that the settling velocities are significantly underestimated in simulations when the settling characteristics of spherical particles are assumed.

Detailed knowledge on the shape and morphology of stratospheric $\mathrm{HNO}_{3}$-containing particles causing denitrification is lacking. Films of $\mathrm{HNO}_{3}$-containing particles near the composition of NAT were characterised by Keyser et al. (1993) under laboratory conditions. The authors report granular micrometre-sized particles forming aggregates. Grothe et al. (2006) analysed the shapes of micrometresized NAT particles under laboratory conditions while inves- tigating the crystallisation kinetics in the presence and absence of ice domains. In the absence of ice domains they obtained plates with diameters of the order of micrometres, whereas needles grew in the presence of ice. Wagner et al. (2005) reported NAD particles obtained in the cloud chamber AIDA (Aerosol Interactions and Dynamics in the Atmosphere), and their spectroscopic measurements are best explained by assuming significantly oblate particles. Hence, large $\mathrm{HNO}_{3}$-containing particles in the stratosphere causing denitrification might be partially composed of aspheric NAD particles, and such particles might act as templates for large NAT particles resulting from transformation of metastable NAD into NAT.

In this work we use measurements of gas-phase $\mathrm{HNO}_{3}$ from the airborne Fourier transform infrared spectrometer MIPAS-STR (Michelson Interferometer for Passive Atmospheric Sounding-STRatospheric aircraft) (Piesch et al., 1996; Woiwode et al., 2012; and references therein) deployed aboard the high-altitude aircraft Geophysica to study denitrification inside the Arctic polar vortex at the end of January 2010. The MIPAS-STR measurements resolve structures resulting from vertical $\mathrm{HNO}_{3}$ redistribution with vertical extensions of the order of $1 \mathrm{~km}$ and horizontal extensions of several tens of kilometres along the flight track and provide information on cloud coverage and temperature.

Utilising simulations performed with CLaMS (Chemical Lagrangian Model of the Stratosphere) (Grooß et al., 2005), we analyse the formation conditions of potential NAT particles sampled in situ by the FSSP-100 during the Geophysica flight on 25 January 2010 . We investigate the sensitivity of vertical $\mathrm{HNO}_{3}$ redistribution to reduced sedimentation velocities of simulated NAT particles and compare the results with the gas-phase $\mathrm{HNO}_{3}$ observations by MIPAS-STR. We consider the hypothesis that the new saturation-dependent parameterisation of heterogeneous NAT nucleation rates applied in CLaMS (Grooß et al., 2014) represents the masses of the largest NAT particles in a realistic way, but that these particles have larger apparent sizes compared to compact spherical particles, e.g. due to aspheric shape and/or noncompact morphology. Furthermore, we analyse sensitivity runs considering temperature biases and modified NAT nucleation rates to estimate the relative impact of these parameters on vertical $\mathrm{HNO}_{3}$ redistribution. Finally, we discuss potential properties of particles involved in denitrification in the context of measured and simulated vertical redistribution of $\mathrm{HNO}_{3}$ and the in situ particle observations.

The combination of a literature review (above) and measurements presented in this study lead to the following assumptions considered in this work: (i) stratospheric $\mathrm{HNO}_{3}$-containing particles might be approximately spherical, but consist of loosely packed aggregates of smaller subunits and therefore have a reduced net particle mass density compared to compact spherical particles, or (ii) these particles might be compact but significantly aspheric (e.g. needle- or disk-shaped). Both assumptions would allow for 
growth of particles with larger maximum sizes (e.g. diameter or length) compared to compact spherical particles, which are often assumed in simulations for simplification. Moreover, in both cases, characteristic reduced settling velocities were expected, altering the vertical redistribution of $\mathrm{HNO}_{3}$.

This study complements a previous study by Woiwode (2013) and takes into account the finalised CLaMS setup for the Arctic winter 2009/10, considering the new saturation-dependent parameterisation of heterogeneous NAT nucleation rates introduced by Grooß et al. (2014) and based on the results by Hoyle et al. (2013).

\section{Campaign, observations and model}

The RECONCILE field campaign was carried out from January until March 2010 and was based in Kiruna, Sweden (von Hobe et al., 2013). Flights of the high-altitude aircraft M-55 Geophysica allowed for probing of the polar vortex with in situ and remote sensing instruments, including the particle probe FSSP-100 and MIPAS-STR. As discussed by Dörnbrack et al. (2012), a strong and cold vortex was formed in the middle of December 2009. The subsequent mid-winter period was exceptionally cold, allowing for the existence of synoptic-scale PSCs until the end of January 2010 and resulting in strong denitrification (Khosrawi et al., 2011). Spaceborne lidar observations indicated PSCs composed of mixtures of NAT and STS (supercooled ternary solutions, composed of $\mathrm{H}_{2} \mathrm{O}, \mathrm{HNO}_{3}$ and $\mathrm{H}_{2} \mathrm{SO}_{4}$ ) inside the Arctic vortex at the end of January 2010 (Pitts et al., 2011).

The measurements from the FSSP-100 (de Reus et al., 2009) are designated for studying the abundances and sizes of micrometre-sized particles. The FSSP-100 is capable of detecting particles with sizes in the range of about 1.05 to $37.5 \mu \mathrm{m}$ based on single forward scattering of laser light. The measurements are usually evaluated considering the Mie theory, assuming approximately spherical particles. Advanced methods (not applied here) allow for the determination of accurate size distributions of aspheric particles, taking into account a priori knowledge on particle shape (Borrmann et al., 2000). Regarding in situ PSC observations during the RECONCILE period and in December 2011, their uncertainties as well as comparisons with collocated observations and simulations we refer to Molleker et al. (2014). For aspheric particles, a broadening of the FSSP size distribution would be expected and the particle volume would be strongly affected by the oversized part. While significant changes in the size distribution already have to be taken into account for particles with aspect ratios of 2 (Borrmann et al., 2000), considerable changes might result for particles with higher aspect ratios. An investigation of this aspect is, however, not within the scope of this work.

The MIPAS-STR limb- and upward-viewing measurements allow for the reconstruction of vertical profiles and cross sections of temperature and trace gas distributions along the flight track and provide information on cloud coverage. Details on the MIPAS-STR sampling and data processing are discussed by Woiwode et al. (2012). Forward calculations and inversion of the MIPAS-STR measurements were carried out using the forward model KOPRA (Karlsruhe Optimized and Precise Radiation transfer Algorithm; Stiller et al., 2002) and the inversion module KOPRAFIT (Höpfner et al., 2001), using the Tikhonov-Phillips regularisation approach (Tikhonov, 1963; Phillips, 1962). Retrievals were carried out utilising a regular vertical $0.5 \mathrm{~km}$ grid in the considered vertical range (grid-spacing increases at higher altitudes). Observations with tangent points lower than $12 \mathrm{~km}$ were omitted to avoid tradeoffs in regularisation. Vertical resolutions given in this context were estimated according to Purser and Huang (1993).

Temperature was retrieved utilising the $\mathrm{CO}_{2}$ signatures in the microwindows from 810.1 to $813.1 \mathrm{~cm}^{-1}$ and 955.6 to $958.5 \mathrm{~cm}^{-1}$. Subsequently, $\mathrm{HNO}_{3}$ was retrieved using the signatures in the microwindow from 866.0 to $870.0 \mathrm{~cm}^{-1}$. Retrieval parameters were the target parameter (temperature or volume mixing ratio) along with wave-numberindependent background continuum, spectral shift and $\mathrm{O}_{3}$ as an additional parameter in the temperature retrieval. Total combined $1 \sigma$ uncertainties were estimated considering uncertainties due to spectral noise, radiometric calibration, spectroscopic line data, line-of-sight knowledge, the adopted $\mathrm{CO}_{2}$ profile (temperature retrieval) and retrieved temperatures $\left(\mathrm{HNO}_{3}\right.$ retrieval). Details on the MIPAS-STR retrieval and its validation are discussed by Woiwode et al. (2012).

No cloud filtering according to Spang et al. (2004) omitting stratospheric spectra with low cloud index values was performed. Practically all stratospheric limb measurements associated with the flight on 25 January 2010 showed cloud index values below 4 , indicating that the flight was carried out inside PSCs. The highest limb views exhibited minimum cloud index values as low as 1.6 as a consequence of continuum absorption by PSC particles. The spectra, however, showed clear trace gas emission signatures suitable for retrievals of atmospheric parameters.

Moderate continuum-like contributions from aerosols are typical for mid-infrared limb observations of the upper troposphere-lower stratosphere (UTLS) region and are considered in the MIPAS-STR data processing by the reconstruction of wave-number-independent background continuum. For the flight on 25 January 2010, spectra with rather low cloud index values were included. Therefore, wavenumber-independent background continuum was inverted logarithmically, resulting in low residuals between the observed and simulated spectra close to the noise level. This approach meets the high dynamical range in the continuum background between spectra associated with different vertical viewing angles, especially at the lower boundaries of PSCs, where the continuum background can decrease sharply. 
Pressure-broadened tropospheric $\mathrm{H}_{2} \mathrm{O}$ and $\mathrm{CO}_{2}$ signatures as described by Höpfner et al. (2004), indicating scattering of tropospheric radiation by PSC particles into the MIPASSTR field of view at high limb views, were not identified. However, the $\mathrm{H}_{2} \mathrm{O}$ retrieval was not exploited for this flight considering the weak intensity of the utilised $\mathrm{H}_{2} \mathrm{O}$ signature under the conditions of this flight and the resulting high uncertainties and low vertical resolution of the retrieval results. Furthermore, it was not clear whether potential weak contributions of scattered tropospheric radiation not obviously identified in the spectra might significantly alter the weak $\mathrm{H}_{2} \mathrm{O}$ signature observed at higher viewing angles.

CLaMS provides a full three-dimensional simulation of both stratospheric chemistry and particle sedimentation based on the Lagrangian concept. Details on the simulation of particle nucleation, growth and sedimentation are given by Grooß et al. (2014, and references therein). The authors successfully applied CLaMS for simulating denitrification in the Arctic winter 2009/10, utilising a new saturation-dependent parameterisation for heterogeneous nucleation rates of NAT (see Hoyle et al., 2013). At temperatures below $T_{\mathrm{NAT}}$, growth and sedimentation of large NAT particles are simulated according to Carslaw et al. (2002).

During the Arctic winter 2009/10, synoptic ice PSCs were frequently observed (Khosrawi et al., 2011; Pitts et al., 2011). NAT nucleation on ice and vice versa was not considered in the simulations associated with this work and might lead to increased condensation of $\mathrm{HNO}_{3}$ and faster downward transport of $\mathrm{HNO}_{3}$ due to larger sizes of the coated particles. On the other hand, such particles would spend less time at certain altitude levels, and therefore less time would be available for the removal of $\mathrm{HNO}_{3}$ from the gas phase through condensation, limiting the potentially resulting additional $\mathrm{HNO}_{3}$ downward transport.

For the particle observations on 25 January 2010 discussed here such coated particles are unlikely, as the associated particle backward trajectories and the observed temperatures shown in the following are not compatible with nucleation and persistence of ice particles due to temperatures above the existence temperature of ice ( $T_{\mathrm{ICE}}$, around $188 \mathrm{~K}$ ). Furthermore, due to the occurrence of a major sudden stratospheric warming around the end of January 2010 (Khosrawi et al., 2011; Pitts et al., 2011; Dörnbrack et al., 2012), the presence of ice particles during the flight on 25 January 2010 appears unlikely.

In the presented simulations, spherical NAT particles with a particle mass density of $1.62 \mathrm{~g} \mathrm{~cm}^{-3}$ (compare Drdla et al., 1993) are assumed in the standard scenario. Horizontal advection is simulated on isentropic levels based on background wind fields provided by ECMWF (European Centre for Medium-Range Weather Forecasts) ERA-Interim reanalyses. Vertical sedimentation of NAT particles is computed considering gravitational settling and the viscosity of air. The sedimentation velocities are calculated according to the Stokes equation (i.e. Pruppacher and Klett, 1997) and un-
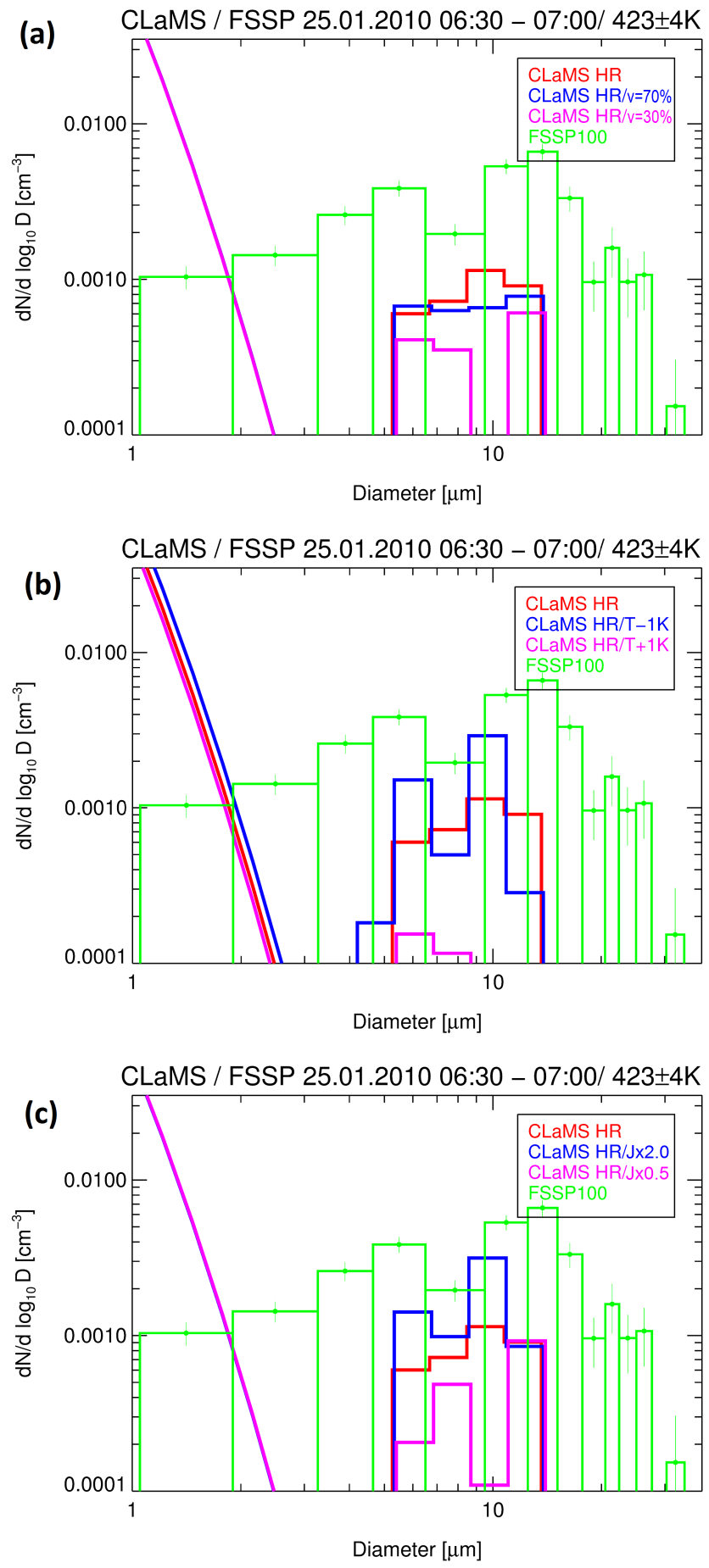

Figure 1. FSSP-100 size distribution derived for the time interval 06:30 to 07:00 UTC of the flight through a PSC on 25 January 2010 (flight altitude $18 \mathrm{~km}$ ) assuming spherical particles. Additionally, the associated size distributions corresponding to the CLaMS reference simulation (CLaMS HR) and alternative scenarios considering reduced settling velocities of NAT particles $(v=70 \%$ and $v=30 \%$, top panel), temperature biases of $\pm 1 \mathrm{~K}(T-1 \mathrm{~K}$ and $T+1 \mathrm{~K}$, middle panel) and modified nucleation rates $(\mathrm{J} \times 0.5$ and $\mathrm{J} \times 2.0$, bottom panel) (compare Sect. 5) are shown. 
der consideration of the Cunningham correction for slip flow for spherical particles (i.e. Müller and Peter, 1992). The horizontal resolution of the considered CLaMS simulations is about $70 \mathrm{~km}$ and the vertical resolution is about $0.7 \mathrm{~km}$.

\section{In situ particle measurements and backward trajectories}

During RECONCILE flights probing potentially NATcontaining PSCs, the FSSP-100 instrument detected large particles with diameters of more than $20 \mu \mathrm{m}$ at temperatures above the frost point under the assumption of spherical particles (von Hobe et al., 2013; Molleker et al., 2014). Figure 1 shows an example FSSP-100 size distribution associated with the vortex flight on 25 January 2010. Two main modes peaking at diameters of about 5.5 and $14 \mu \mathrm{m}$ with very high number densities of about 0.004 and $0.007 \mathrm{~cm}^{-3}$ can be identified. Particles with extremely large diameters of more than $20 \mu \mathrm{m}$ are found. The maximum number density of the second mode is about a factor of 5 higher than the maximum of the large NAT mode reported by Fahey et al. (2001) peaking at $14.5 \mu \mathrm{m}$. The shown representation indicates differential number densities. In this representation, the large NAT mode discussed by Fahey et al. (2001) peaks at about $0.0014 \mathrm{~cm}^{-3}$ (compare Molleker et al., 2014). Assuming spherical particles, the $\mathrm{HNO}_{3}$ content of the complete size distribution is estimated to be equivalent to about $11.5 \mathrm{ppbv}$ of gas-phase $\mathrm{HNO}_{3}$ considering an ambient temperature of $195 \mathrm{~K}$ and a pressure of $60 \mathrm{hPa}$. Thereby, $11 \mathrm{ppbv}$ would correspond to the size bins larger than $9.5 \mu \mathrm{m}$.

Figure 1 shows also the associated size distribution corresponding to the CLaMS reference scenario assuming compact spherical particles (CLaMS HR). The particle mode situated between about 5 and $11 \mu \mathrm{m}$ and causing the simulated denitrification is by a factor of 2 to 6 lower compared to the observed number densities in this size range. The largest particles observed with sizes above $11 \mu \mathrm{m}$ are not reproduced by the simulation. One possible (partial) explanation for the observed discrepancy might be that observed NAT particles have masses close to the simulated values, but larger apparent sizes (diameter or length) compared to compact spherical particles due to non-compact morphology and/or aspheric shapes. The small particle mode below $2 \mu \mathrm{m}$ corresponds to simulated STS droplets. The high number density mode of small NAT particles described by Grooß et al. (2005) was not considered in the discussed simulations. Also shown in Fig. 1 are the corresponding size distributions obtained when alternative model setups are applied (see Sect. 5). While the shape of the NAT mode is modified in the alternative scenarios and the $T+1 \mathrm{~K}$ scenario hardly produces any NAT particles capable of denitrification for this flight, these scenarios also fail to reproduce particles with masses larger than that of compact spherical particles with diameters of 11 to $12 \mu \mathrm{m}$.

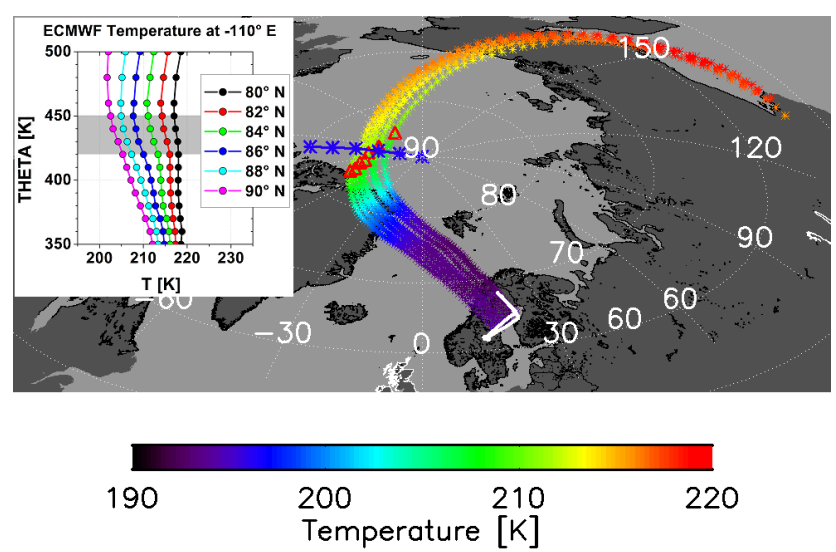

Figure 2. CLaMS particle backward trajectories coloured according to temperature (asterisks) for simulated NAT particles with diameters between 5 and $11 \mu \mathrm{m}$ associated with the Geophysica flight on 25 January 2010. Prior to the nucleation events the particle trajectories are continued by air mass trajectories for the air volumes where nucleation occurred. The flight track of the Geophysica is indicated by a solid white line. Positions where the trajectories reach $210 \mathrm{~K}$ are marked by red triangles. Blue asterisks connected by a blue line indicate the positions of the temperature profiles shown in the inset. The grey shading in the inset indicates the potential temperature (THETA) levels corresponding to the vertical range of about $18 \mathrm{~km}$ to $20 \mathrm{~km}$ in the model domain (from Woiwode PhD thesis, 2013, with modifications).

For eight representative particles with diameters between 5 and $11 \mu \mathrm{m}$ that were generated by the reference simulation, the corresponding sedimentation backward trajectories were reconstructed. Prior to the nucleation times, the trajectories were continued by air mass trajectories in order to further study the history of the air masses where the nucleation occurred. The obtained trajectories are presented in Fig. 2 together with the flight track of the Geophysica on 25 January 2010. The extracted trajectories beginning at 07:00 UTC show the following characteristics: (i) the trajectories remain compact during the entire interval of about 4.5 days considered, indicating the absence of significant shear in the flow of the overlaying air masses passed by the individual particles at different times as a consequence of their different settling velocities. (ii) All trajectories approach $T_{\mathrm{NAT}}$ (typically between 194 and $198 \mathrm{~K}$, depending on the actual partial pressures of $\mathrm{H}_{2} \mathrm{O}$ and $\mathrm{HNO}_{3}$ ) around the north-east coast of Greenland about 2 days prior to the FSSP-100 measurements, resulting in growth of the simulated compact spherical particles to maximum diameters of not more than $11 \mu \mathrm{m}$ in the CLaMS domain. (iii) Going further back in time, the temperatures of the associated air parcels further increase. Temperatures of $210 \mathrm{~K}$ (red triangles) are reached about 2.8 days prior to the in situ particle observations. (iv) Temperature profiles between 80 and $90^{\circ} \mathrm{N}$ perpendicular to the locations where the trajectories reach about $210 \mathrm{~K}$ (blue asterisks connected by line) show all temperatures that are well above 


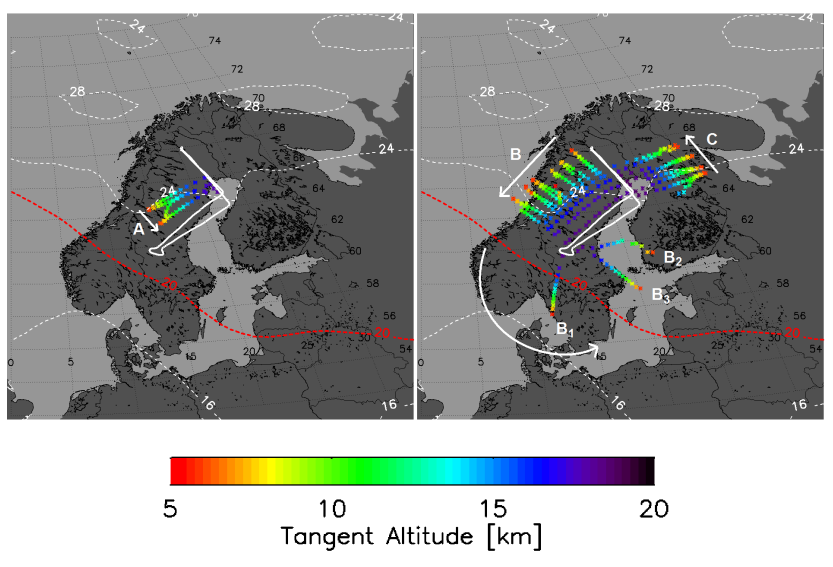

Figure 3. Tangent points associated with the MIPAS-STR measurements during the PSC flight on 25 January 2010 colour-coded according to altitude (asterisks). The location of the polar vortex is indicated by isolines of potential vorticity (dotted white lines, values in PVU) at the potential temperature level of $430 \mathrm{~K}$ (approximately $18 \mathrm{~km}$ ) as extracted from the ECMWF ERA-Interim reanalysis. The vortex edge is indicated according to Nash et al. (1996) by the red dotted line (from Woiwode PhD thesis, 2013, with modifications).

$T_{\mathrm{NAT}}$ in the vertical range considered (inset in Fig. 2). Considering that the ambient regions were also characterised by temperatures above $T_{\mathrm{NAT}}$ and the compactness of the trajectories for particles with diameters between 6 and $11 \mu \mathrm{m}$ it appears unlikely that larger particles have entered the locations of the discussed particle observations along very different trajectories. Note that temperature fluctuations along the trajectories that are not considered by the model might also influence the size distribution of real NAT particles in the stratosphere. However, considering the steady increase of the temperatures along the discussed trajectories towards temperatures well above $T_{\mathrm{NAT}}$, temperature fluctuations of a few $\mathrm{K}$ are unlikely to explain the large particle sizes observed. Furthermore, the size distribution of the scenario considering a negative temperature bias of $1 \mathrm{~K}$ (Fig. 1; see also Sect. 5) does not change the simulated maximum sizes notably.

In summary, for the flight on 25 January 2010 the CLaMS simulations indicate growth of compact spherical particles with maximum diameters not larger than 11 to $12 \mu \mathrm{m}$ as a consequence of limited growing time. In contrast, the FSSP-100 observations shown in Fig. 1 indicate potential NAT particles with maximum sizes larger than $20 \mu \mathrm{m}$.

\section{MIPAS-STR measurements of temperature, PSC coverage and gas-phase $\mathrm{HNO}_{3}$}

For the flight on 25 January 2010 (take-off 05:50 UTC and landing 09:19 UTC in Kiruna, Sweden), the flight track of the Geophysica and the horizontal distribution of the tangent points associated with the MIPAS-STR observations are shown in Fig. 3. Practically all MIPAS-STR observations

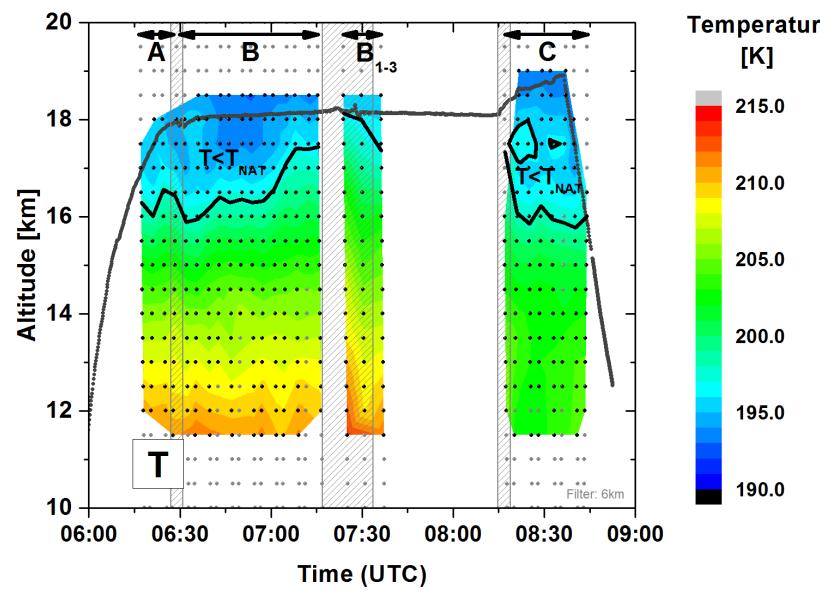

Figure 4. Vertical cross section of temperature retrieved from the MIPAS-STR observations associated with the flight on 25 January 2010. The solid black lines indicate levels where temperatures retrieved from MIPAS-STR are equal to calculated $T_{\text {NAT }}$ (see text). The flight altitude of the Geophysica is indicated by a solid grey line. The retrieval grid is indicated by black dots. Grey dots were excluded from interpolation due to low vertical resolution. Grey hatched areas indicate turns performed by the Geophysica, where the retrieval results are characterised by increased uncertainties (from Woiwode $\mathrm{PhD}$ thesis, 2013, with modifications).

were located inside the polar vortex. The tangent points of the MIPAS-STR observations subsequently covered the regions labelled A, B and C. During the turn-around at 07:30 UTC, further scans, named $\mathrm{B}_{1}, \mathrm{~B}_{2}$ and $\mathrm{B}_{3}$, were performed. $\mathrm{Be}-$ tween the scans $\mathrm{B}_{3}$ and $\mathrm{C}$, sampling was interrupted as the instrument was pointing towards the rising sun.

In Fig. 4 the retrieved vertical distribution of temperature is shown for the flight the on 25 January 2010. The interval of the FSSP-100 measurements (Fig. 1) and the starting positions of the particle backward trajectories (Fig. 2) correspond to the interval between 06:30 and 07:00 UTC in section B. Also shown is the level where the retrieved temperatures from MIPAS-STR are equal to the existence temperature of NAT (at higher altitudes the retrieved temperatures are below $\left.T_{\mathrm{NAT}}\right) . T_{\mathrm{NAT}}$ and the $T_{\mathrm{NAT}}$ line were calculated considering the MIPAS-STR retrieval results of $\mathrm{HNO}_{3}$ (see below, typical vertical resolution about $1-2 \mathrm{~km}$ ) and temperature (typical vertical resolution about $2-3 \mathrm{~km}$ ) in combination with a smoothed vertical profile of $\mathrm{H}_{2} \mathrm{O}$ constructed from the in situ observations by FLASH-A (Khaykin et al., 2013) during the ascent and descent phase of the discussed flight (i.e. $\mathrm{H}_{2} \mathrm{O}$ volume mixing ratios of 4.2 ppmv below $17.5 \mathrm{~km}$ and values within 4.2 to $5 \mathrm{ppmv}$ at higher altitudes). Thermodynamic parameters for the calculation of $T_{\mathrm{NAT}}$ were taken from Hanson and Mauersberger (1988). The absolute values obtained for $T_{\mathrm{NAT}}$ are typically about $198 \mathrm{~K}$.

Temperatures below $T_{\mathrm{NAT}}$ and above $T_{\mathrm{ICE}}$ are found around flight altitude during the entire flight. In sections A, B and C, temperatures approach $T_{\mathrm{NAT}}$ mainly around an 


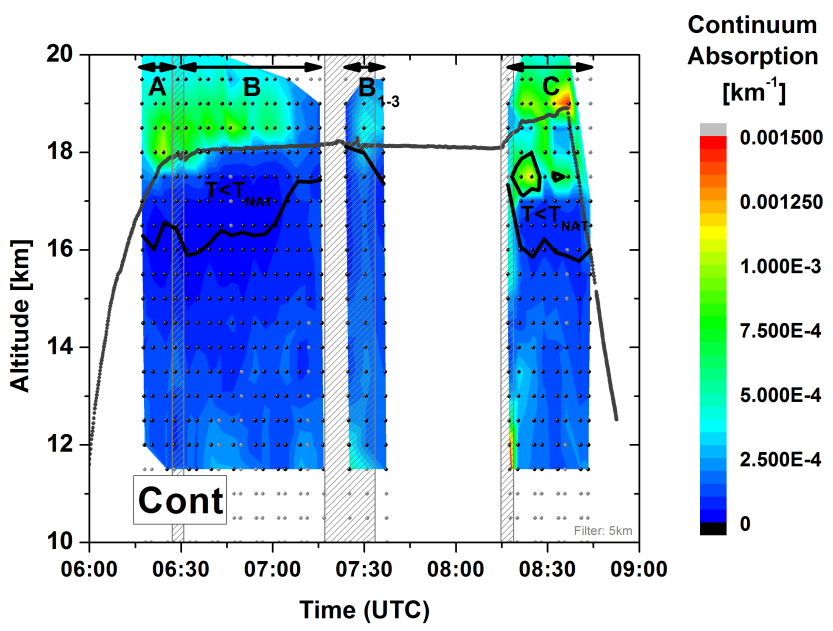

Figure 5. Vertical cross section of continuum absorption retrieved from the MIPAS-STR observations associated with the flight on 25 January 2010. Enhanced continuum absorption indicates cloud/aerosol particles. Other parameters as in Fig. 4 (from Woiwode $\mathrm{PhD}$ thesis, 2013, with modifications).

altitude of $16 \mathrm{~km}$. Warmer temperatures are found between the end of section B (after 07:00 UTC) and the beginning of section $\mathrm{C}$, with the $T_{\mathrm{NAT}}$ line rising up to $18 \mathrm{~km}$. In section $\mathrm{C}$, two spots situated around $17.5 \mathrm{~km}$ show temperatures slightly above the calculated $T_{\mathrm{NAT}}$ (i.e. less than $1 \mathrm{~K}$ ).

Figure 5 shows the corresponding cross section of retrieved wave-number-independent continuum absorption for the 810.1 to $813.1 \mathrm{~cm}^{-1}$ microwindow associated with the temperature retrieval. Enhanced continuum absorption qualitatively indicates the presence of broadband absorbers such as cloud and aerosol particles. Along almost the entire flight track, significantly increased continuum absorption indicates the presence of PSC particles around flight altitude and above. An extended maximum is found in the section between 06:15 and 07:10 UTC, with a sharp contrast to low continuum absorption indicating the absence of continuum absorbers below about 17.5 to $18.0 \mathrm{~km}$. In the last part of the flight between 08:15 and 08:45 UTC (section C), enhanced continuum absorption is found at altitudes higher than $17 \mathrm{~km}$. Local minima around 18.0 to $18.5 \mathrm{~km}$ suggest different PSC layers.

In the vertical regions where enhanced continuum absorption indicates the presence of PSCs, temperatures below $T_{\mathrm{NAT}}$ are found. The only exceptions are the two spots around $17.5 \mathrm{~km}$ in the section with retrieved temperatures slightly above $T_{\mathrm{NAT}}$, which are probably due to the limited vertical resolution and uncertainties of the MIPAS-STR retrieval results or effects from horizontal gradients along the viewing direction. For example, a PSC layer might be located in a colder region along the viewing direction, while warmer temperatures in other sections along the viewing direction might lead to a warmer net temperature retrieved. Furthermore, the

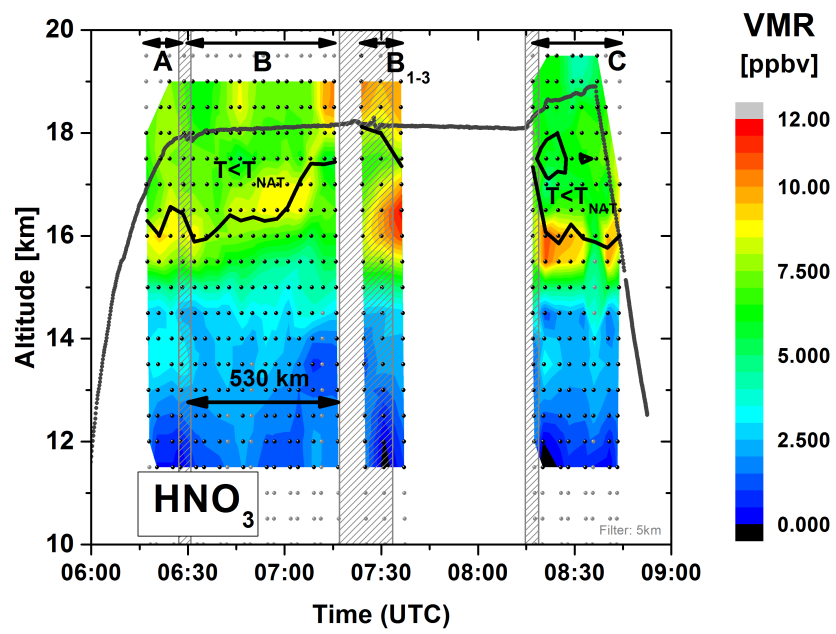

Figure 6. Vertical cross section of gas-phase $\mathrm{HNO}_{3}$ retrieved from the MIPAS-STR observations associated with the flight on 25 January 2010. Other parameters as in Fig. 4 (from Woiwode $\mathrm{PhD}$ thesis, 2013, with modifications).

values obtained for $T_{\mathrm{NAT}}$ are sensitive to potential local variations in the mixing ratios of $\mathrm{H}_{2} \mathrm{O}$ (see Khaykin et al., 2013) and retrieved $\mathrm{HNO}_{3}$. The sensitivity of calculated $T_{\mathrm{NAT}}$ towards variations in the mixing ratios of these gases is discussed below (see Fig. 7). It can serve as an estimate for the amplitudes of variations in $T_{\mathrm{NAT}}$ as well as the delta between temperature and $T_{\mathrm{NAT}}$ due to horizontal gradients and/or local temperature fluctuations.

Figure 6 shows the associated vertical distribution of $\mathrm{HNO}_{3}$ along the flight track retrieved from the MIPAS-STR measurements. The horizontal extent of section B is indicated for guidance. Local maxima of $\mathrm{HNO}_{3}$ are found at altitudes ranging from 15.5 to $17.0 \mathrm{~km}$. In sections A and $\mathrm{B}$, maximum volume mixing ratios of $\mathrm{HNO}_{3}$ of $8.5 \mathrm{ppbv}$ are found, compared to minimum values below $6.5 \mathrm{ppbv}$ around in this vertical region. The maxima in section $B_{1}$ to $B_{3}$ peak at about $10 \mathrm{ppbv}$ above flight altitude and $11 \mathrm{ppbv}$ at $16.5 \mathrm{~km}$, compared to minimum values around $7 \mathrm{ppbv}$ in between. Another strong maximum is observed in section $\mathrm{C}$, peaking at 15.5 to $16 \mathrm{~km}$ altitude, and shows enhanced $\mathrm{HNO}_{3}$ mixing ratios of $10.5 \mathrm{ppbv}$ compared to minimum values of less than $6 \mathrm{ppbv}$ around. The local maxima show excess $\mathrm{HNO}_{3}$ resulting from renitrification, with denitrified air masses above (compare also Sect. 5). While denitrified layers present at higher altitudes are not covered by the MIPAS-STR observations, denitrification at higher altitudes at that stage of the polar winter was confirmed by Aura/MLS observations (Khosrawi et al., 2011, Fig. 3). The resolved vertical thickness of the local $\mathrm{HNO}_{3}$ maxima in Fig. 5 is of the order of $1 \mathrm{~km}$. The locations of the $\mathrm{HNO}_{3}$ maxima show considerable coincidence with the regions where the retrieved temperatures approach $T_{\mathrm{NAT}}$, supporting the evaporation of NAT particles around these altitudes. 

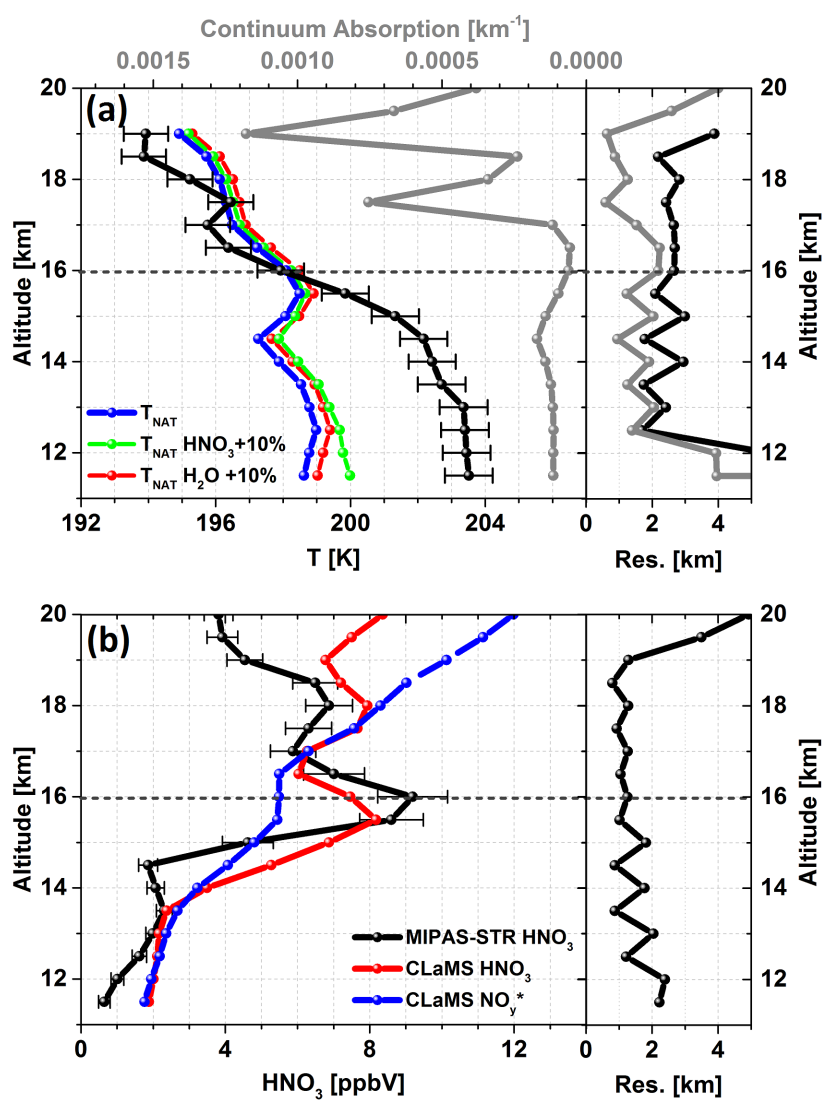

Figure 7. Upper panel (a): temperature $(T)$ and wave-numberindependent background continuum absorption retrieved from MIPAS-STR for the limb scan at 08:32 UTC. Corresponding profiles of $T_{\text {NAT }}$ were calculated considering retrieved $\mathrm{HNO}_{3}$ in combination with the smoothed $\mathrm{H}_{2} \mathrm{O}$ profile from FLASH-A (alternative profiles considering increased $\mathrm{HNO}_{3}$ and $\mathrm{H}_{2} \mathrm{O}$ are also indicated). Lower panel (b): corresponding profiles of $\mathrm{HNO}_{3}$ retrieved from MIPAS-STR and extracted from the CLaMS reference scenario together with simulated passive $\mathrm{NO}_{\mathrm{y}}^{*}$. Error bars on the left-hand side of the panels correspond to estimated total $1 \sigma$ uncertainties of the retrieval results. "Res." on the right-hand side of the panels corresponds to vertical resolution of the retrieved profiles. Grey dashed lines indicate altitude with $T$ equal to $T_{\mathrm{NAT}}$.

Typical profiles retrieved from the MIPAS-STR observations, estimated uncertainties and vertical resolutions are shown in Fig. 7. For the temperature retrieval (Fig. 7a), typical vertical resolutions of 2 to $3 \mathrm{~km}$ were obtained, while the estimated $1 \sigma$ uncertainties are typically lower than $0.8 \mathrm{~K}$. For continuum absorption, two distinct maxima are found, peaking at 17.5 and $19 \mathrm{~km}$ and indicating two different PSC layers. The typical vertical resolution obtained is about 1 to $2 \mathrm{~km}$. We mention that the alignment of the PSC layers in the horizontal direction along the line of sight is uncertain.

Also shown is the corresponding profile of calculated $T_{\mathrm{NAT}}$ considering the nominal values for $\mathrm{HNO}_{3}$ (MIPAS-STR) and $\mathrm{H}_{2} \mathrm{O}$ (FLASH-A smoothed) together with the same profiles considering biases of $+10 \%$ for the volume mixing ratios of these gases. The retrieved temperature is equal to $T_{\mathrm{NAT}}$ at about $16 \mathrm{~km}$ altitude, and supersaturated conditions are found above. The continuum absorption maximum associated with the lower PSC layer peaks $1.5 \mathrm{~km}$ above, indicating that supersaturated conditions are also present at regions with weak continuum absorption (i.e. cloud-free). The retrieved temperatures above $16 \mathrm{~km}$ are below $T_{\mathrm{NAT}}$ by up to about $2 \mathrm{~K}$. The shifts in $T_{\mathrm{NAT}}$ due to enhanced $\mathrm{HNO}_{3}$ and $\mathrm{H}_{2} \mathrm{O}$ are small and comparable to the uncertainties of the retrieved temperatures.

Figure $7 \mathrm{~b}$ shows the retrieved profiles of $\mathrm{HNO}_{3}$ with typical $1 \sigma$ uncertainties of about $10 \%$ and a high typical vertical resolution of about $1 \mathrm{~km}$ above $15 \mathrm{~km}$ and 1 to $2 \mathrm{~km}$ below. The corresponding $\mathrm{HNO}_{3}$ profile from the CLaMS standard scenario is shown for comparison together with simulated passive $\mathrm{NO}_{\mathrm{y}}^{*}$ (i.e. considering no condensation and vertical redistribution of $\mathrm{HNO}_{3}$ ). The comparison shows that the maxima observed by MIPAS-STR peaking at about 16 and $18 \mathrm{~km}$ are reproduced well. Note that vertical fine structures of the order of $1 \mathrm{~km}$ and localised profiles are considered. The simulated peak values differ each by about $1 \mathrm{ppbv}$ from the retrieved values and the lower maximum is significantly shifted towards lower altitudes by 0.5 to $1.0 \mathrm{~km}$. Considerably higher simulated $\mathrm{HNO}_{3}$ mixing ratios compared to the observations are found above $18.5 \mathrm{~km}$. While even several fine structures are reproduced well by the simulation, the observed stronger discrepancies from the observations (especially at higher altitudes) are primarily attributed to the complex PSC scenery with significant amounts of $\mathrm{HNO}_{3}$ being condensed.

In summary, (i) the in situ observations of large potential NAT particles, (ii) the retrieved vertical cross section of continuum absorption indicating extended PSC coverage along the flight track, (iii) temperatures below $T_{\mathrm{NAT}}$ and above $T_{\mathrm{ICE}}$ around the flight altitude, and (iv) the observed $\mathrm{HNO}_{3}$ maxima vertically coinciding with the $T_{\mathrm{NAT}}$ line suggest an ongoing denitrification process with NAT particles being involved. Furthermore, PSCs classified as NAT were observed by lidar from Esrange (Kiruna, Sweden) on 24 January 2010 at around $18 \mathrm{~km}$ altitude, supporting a composition of NAT at these altitudes at the day before the discussed Geophysica flight was performed (P. Achtert, personal communication, 2014; no lidar observations were available for 25 January 2010).

The retrieved continuum distribution shows no significant enhancement within typically 1.0 to $1.5 \mathrm{~km}$ above the $T_{\mathrm{NAT}}$ line and where the $\mathrm{HNO}_{3}$ maxima are found. Considering the in situ observations of large potential NAT particles with sizes of tens of micrometres (Fig. 1) at the lower edge of the PSC (Fig. 5, 06:30 to 07:00 UTC) and potentially capable of settling several hundreds of metres per day (compare Pruppacher and Klett, 1997; Fahey et al., 2001), the scenario shown would be compatible with very few large NAT particles falling out of a more dense PSC around flight altitude (e.g. mixed-phase PSC containing NAT and potentially 
Table 1. CLaMS scenarios considering reduced settling velocities for simulated NAT particles ( $v$ : velocity; $D$ : diameter; $\rho$ : particle mass density; $h$ : height; AR: aspect ratio). All indicated example particles have the same mass but different relative settling velocities.

\begin{tabular}{lr|rrr}
\hline \multicolumn{2}{c|}{ Model setup } & \multicolumn{3}{c}{ Corresponding particle properties } \\
\hline Scenario & $\begin{array}{r}\text { Relative } \\
\text { settling speed }\end{array}$ & $D[\mu \mathrm{m}] / \rho\left[\mathrm{g} \mathrm{cm}^{-3}\right]$ & $\begin{array}{r}\text { Needle-shaped } \\
h[\mu \mathrm{m}] / \mathrm{AR}\end{array}$ & $\begin{array}{r}\text { Disk-shaped* } \\
D[\mu \mathrm{m}] / \mathrm{AR}\end{array}$ \\
\hline$v=100 \%$ & 1.00 & $10 / 1.62$ & - & - \\
$v=70 \%$ & 0.7 & $14 / 0.56$ & $34 / 7.6$ & $11 / 0.44$ \\
$v=50 \%$ & 0.5 & $20 / 0.20$ & $68 / 21.9$ & $21 / 0.07$ \\
$v=30 \%$ & 0.3 & $33 / 0.04$ & $168 / 84.5$ & $38 / 0.01$ \\
\hline
\end{tabular}

* Assuming horizontally oriented disks (see text).

STS and giving rise to significant continuum absorption) and evaporating at altitudes where temperatures approach $T_{\mathrm{NAT}}$. This is furthermore supported by the fact that the FSSP100 started detecting potential NAT particles already around $17 \mathrm{~km}$ altitude during the ascent phase, which is below the PSC detected by MIPAS-STR in this region (compare Fig. 5, section $\mathrm{A}$ and the beginning of section $\mathrm{B}$ ).

\section{CLaMS simulations and comparison with MIPAS-STR}

The analysis described in the following investigates the impact of reduced settling velocities of NAT particles on the denitrification process. Reduced settling velocities are expected, for instance, for (i) approximately spherical NAT particles with low mass density and (ii) aspheric NAT particles, which might explain the in situ observations of large particles on 25 January 2010. Different CLaMS scenarios considering the NAT particle settling velocities reduced by constant factors were carried out, and the impact on the fingerprint in the simulated vertical redistribution of $\mathrm{HNO}_{3}$ was analysed. The reference simulation and the scenarios considering reduced settling velocities of NAT particles are summarised in Table 1 . The reference simulation is the scenario $v=100 \%$ (settling velocities of simulated NAT particles not modified; " $v$ " stands for relative settling velocity). Three alternative setups with the settling velocities of the simulated NAT particles multiplied by constant factors of $0.7(v=70 \%), 0.5$ $(v=50 \%)$ and $0.3(v=30 \%)$ were carried out. Furthermore we investigated the relative impact of temperature biases of $\pm 1 \mathrm{~K}$ (scenarios $T+1 \mathrm{~K}$ and $T-1 \mathrm{~K}$ ) and the NAT nucleation rates multiplied by factors of 0.5 and 2.0 (scenarios $\mathrm{J} \times 0.5$ and $\mathrm{J} \times 2.0$ ) on vertical $\mathrm{HNO}_{3}$ redistribution.

In the following, MIPAS-STR measurements of vertical redistribution of $\mathrm{HNO}_{3}$ are compared with the results from the different CLaMS scenarios for the two vortex flights on 25 January 2010 and 30 January 2010. As discussed above, the first flight was carried under the conditions of synopticscale PSCs and probably ongoing vertical $\mathrm{HNO}_{3}$ redistribution. The second flight was performed on 30 January 2010

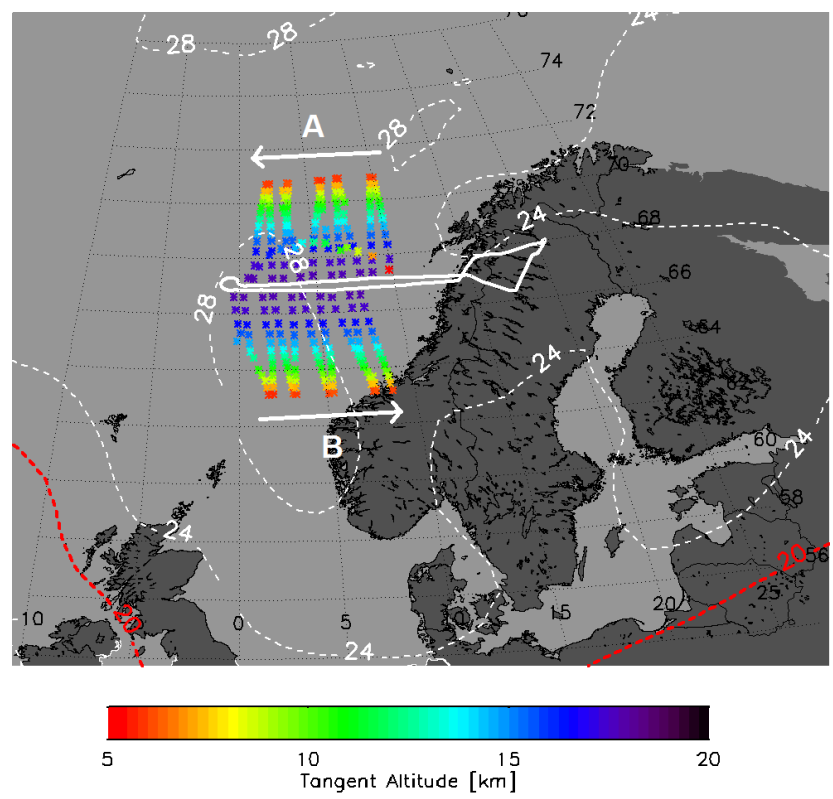

Figure 8. MIPAS-STR observations and meteorological situation during the flight on 30 January 2010 under conditions free of PSCs. Compare Fig. 3 (from Woiwode PhD thesis, 2013, with modifications).

under conditions free of PSCs. The flight track and distribution of the MIPAS-STR tangent points are shown in Fig. 8. The observations suitable for processing were between 07:30 and 09:00 UTC and were situated well within the polar vortex according to the definition of Nash et al. (1996) at the potential temperature level of about $430 \mathrm{~K}$ (determined from the ECMWF ERA-Interim reanalysis). The cloud index values (not shown) of the MIPAS-STR observations associated with this flight indicate cloud-free conditions at stratospheric altitudes (i.e. values higher than 4 according to Spang et al., 2004), and the retrieved temperatures (not shown) are above calculated $T_{\mathrm{NAT}}$.

As the horizontal resolution along the viewing direction and the vertical resolution of the MIPAS-STR observations is somewhat lower compared to the CLaMS simulations, local 
(a)

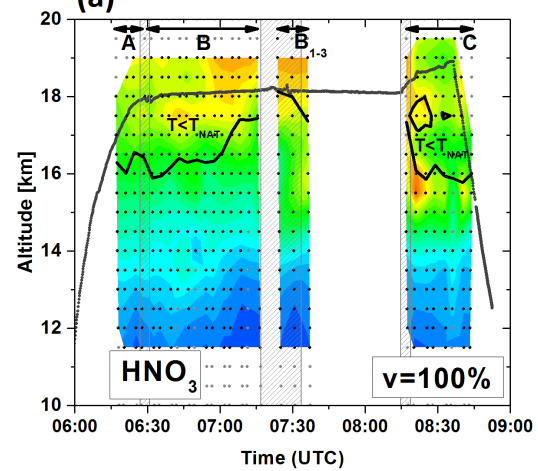

(c)

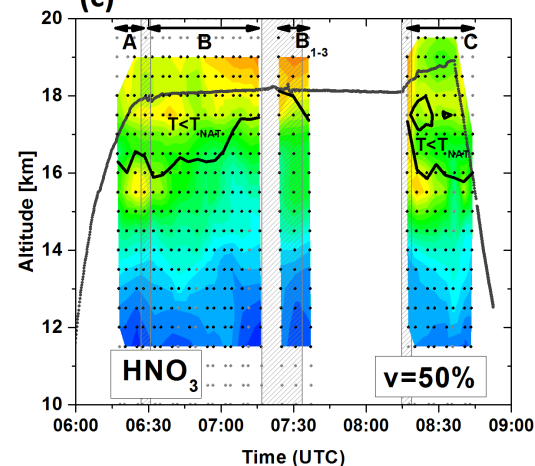

(e)

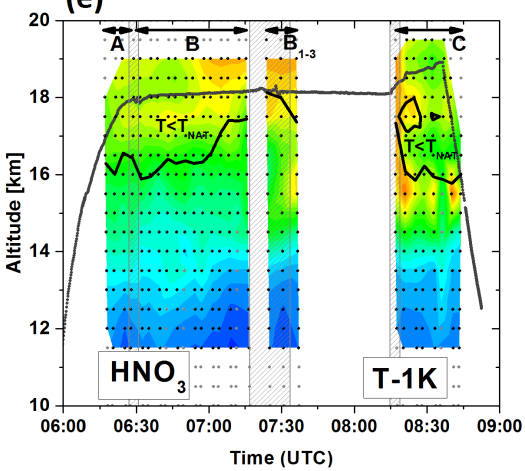

(g)

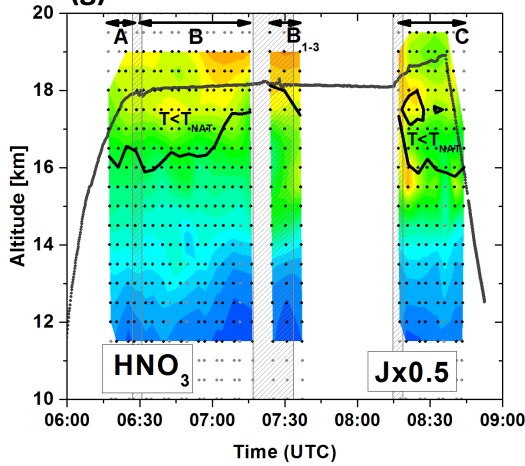

(b)

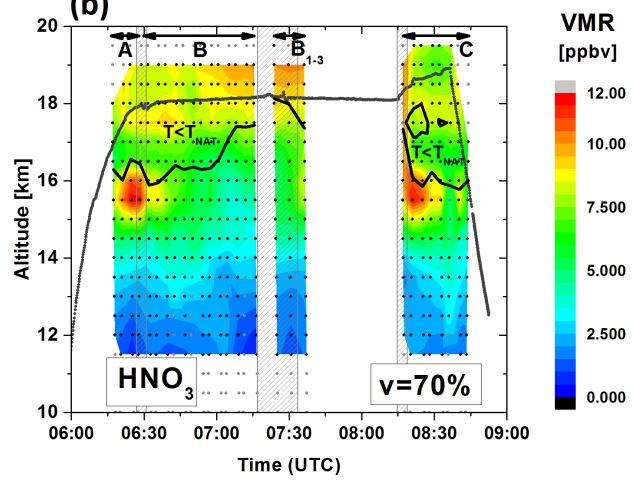

(d)
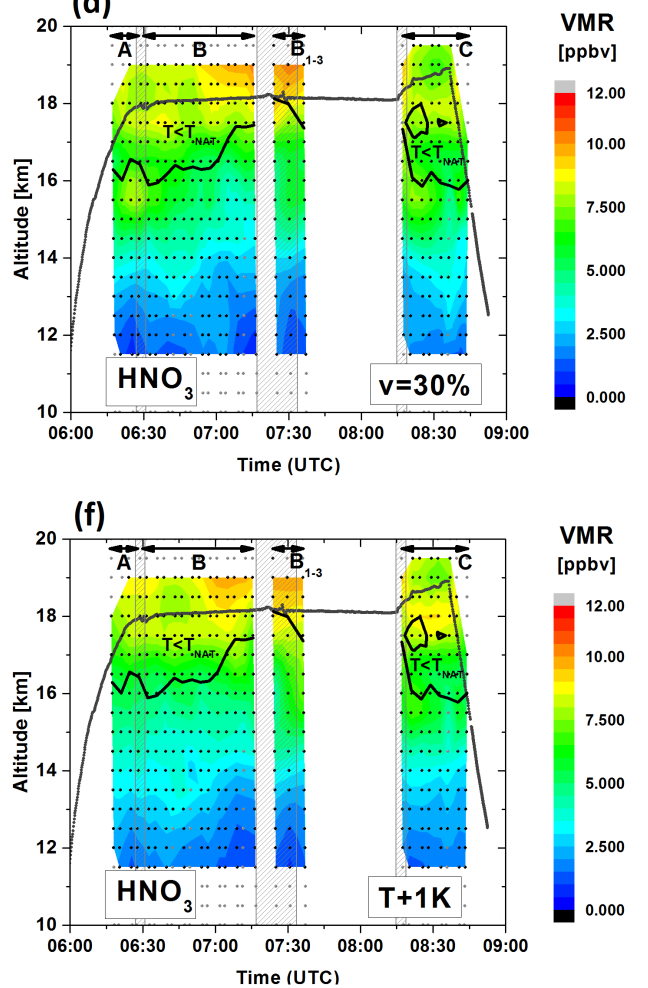

(h)

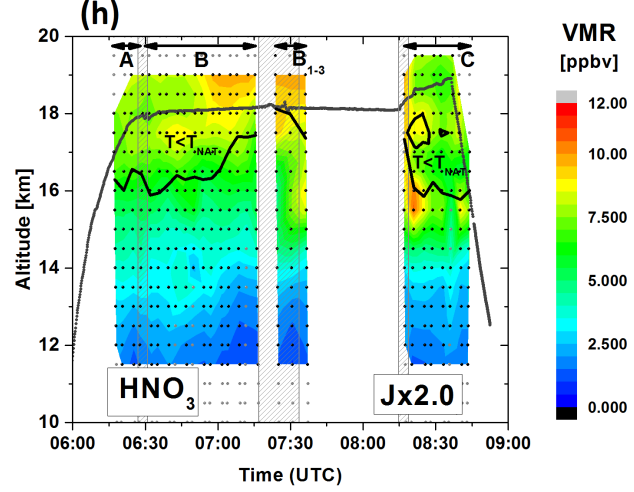

Figure 9. Vertical cross sections of gas-phase $\mathrm{HNO}_{3}$ extracted from the CLaMS reference simulation $(v=100 \%)$, scenarios considering reduced settling velocities of NAT particles $(v=70 \%, v=50 \%$ and $v=30 \%)$, temperature biases of $\pm 1 \mathrm{~K}(T-1 \mathrm{~K}$ and $T+1 \mathrm{~K})$ and modified nucleation rates $(\mathrm{J} \times 0.5$ and $\mathrm{J} \times 2.0)$ for the PSC flight on 25 January 2010. Other parameters (including $T_{\mathrm{NAT}}$ line) as in Fig. 6 . 
atmospheric fine structures are resolved to a lesser degree. To consider for effects from atmospheric inhomogeneities along the viewing direction of MIPAS-STR, the CLaMS results were extracted (i) directly at the individual virtual tangent points (i.e. spatial interpolation of the finer retrieval grid onto the tangent point geolocations) of the MIPAS-STR measurements and the same positions also shifted (ii) towards and (iii) away from the observer by the half-distance between observer and nominal virtual tangent point, weighted with a ratio of $3: 1: 1$. Measurements at and above flight altitude were also smoothed horizontally in a similar manner by extracting the model results at the observer coordinates and two further positions along the viewing direction. This simple approach allows for a useful approximation of the limited horizontal resolution of an infrared limb sounder along the viewing direction, which typically increases from several tens to a few hundreds of kilometres from the observer altitude towards lower altitudes (compare Ungermann et al., 2012). Furthermore, the vertical smoothing inherent to the MIPASSTR measurements was taken into account by smoothing the resulting CLaMS profiles with the averaging kernels of the corresponding individual profiles retrieved from the MIPASSTR observations according to Rodgers (2000).

\subsection{Vertical cross sections}

Figure 9 shows the vertical cross section of $\mathrm{HNO}_{3}$ obtained from the CLaMS reference scenario $v=100 \%$ (Fig. 9a) along with scenarios considering reduced settling velocities of the simulated NAT particles (Fig. 9b-d), temperature biases (Fig. 9e-f) and modified NAT nucleation rates (Fig. 9g-h). The cross sections correspond to the MIPASSTR observations shown in Fig. 6. The major structures indicated by the MIPAS-STR observations are reproduced by all scenarios to a high degree. While comparably low mixing ratios are found around flight altitude in section $\mathrm{A}$, the first part of section $B$, and section $C$, enhanced values are found at these altitudes at the end of section $\mathrm{B}$ and for the scans $\mathrm{B}_{1}$ to $\mathrm{B}_{3}$.

The strong $\mathrm{HNO}_{3}$ maximum indicated by the MIPAS-STR observations at 15.5 to $16.0 \mathrm{~km}$ in section $\mathrm{C}$ is reproduced best by the scenario $v=70 \%$. To a lesser extent it is also reproduced by the scenarios $v=100 \%, v=50 \%$ and $T-1 \mathrm{~K}$, as well as the scenarios with modified nucleation rates. In contrast, this structure is practically missing in the scenarios $v=30 \%$ and $T+1 \mathrm{~K}$. The faint maximum indicated by MIPAS-STR around $16 \mathrm{~km}$ in section A can be identified to a different extent only in the scenarios considering reduced settling velocities. Another strong maximum indicated by MIPAS-STR in scans $B_{1}$ to $B_{3}$ around 16.0 to $17 \mathrm{~km}$ and the faint maximum in the middle of section $\mathrm{B}$ around 16.5 to $17 \mathrm{~km}$ are only mariginally reproduced or not at all by the individual simulations. Further weak local maxima and substructures produced by the simulation cannot be attributed one to one to structures identified in the MIPAS-STR obser-

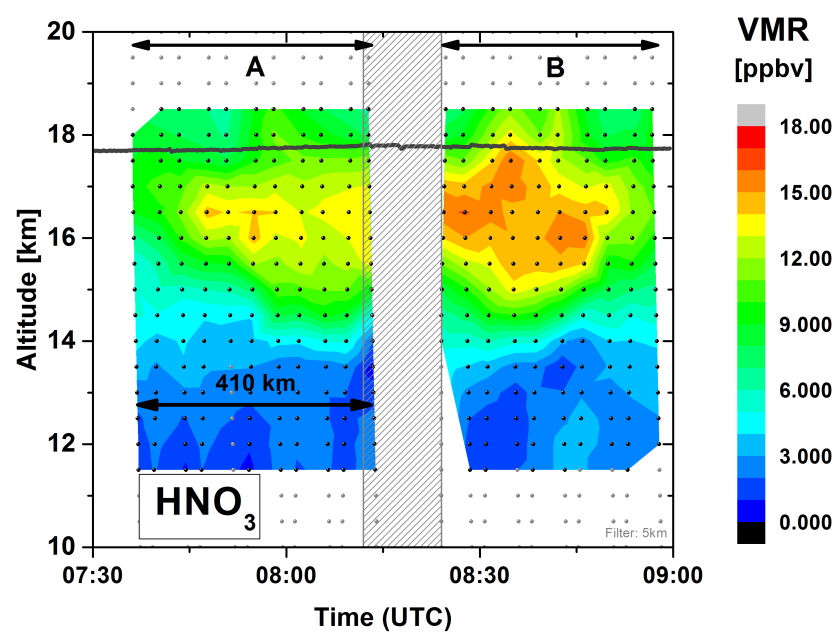

Figure 10. Vertical cross section of gas-phase $\mathrm{HNO}_{3}$ retrieved from the MIPAS-STR observations associated with the flight on 30 January 2010. Compare Fig. 6 (from Woiwode PhD thesis, 2013, with modifications).

vations and vice versa and are consequences of differences in the model domain (e.g. limited spatial resolution and accuracy of the temperature and wind background fields used in the simulations as well as the way fine-scale structures are generated through the mixing parameterisation) and the atmosphere as observed by the instrument (e.g. smoothing characteristics along line of sight and uncertainties of the measurements).

Figure 10 shows the vertical cross section of $\mathrm{HNO}_{3}$ retrieved from the MIPAS-STR observations associated with the flight on 30 January 2010 under conditions free of PSCs. Along the subsequent flight legs with the instrument looking towards the north and south, strong $\mathrm{HNO}_{3}$ maxima are found below flight altitude (sections $\mathrm{A}$ and $\mathrm{B}$, respectively). The structures peak around $16 \mathrm{~km}$ altitude and show maximum mixing ratios as high as $16 \mathrm{ppbv}$ (note that the range of the colour coding is different compared to Figs. 6 and 9).

The corresponding cross sections extracted from the individual CLaMS simulations are shown in Fig. 11. All scenarios essentially reproduce the $\mathrm{HNO}_{3}$ maxima to the north and south of the flight path. The amplitudes, vertical locations and the fine-scale patterns of these features are reproduced to a different extent by the individual scenarios. Besides the overall good agreement for the major structures, the scenarios considering reduced settling velocities result in a tailing of the maxima at the begin of section $\mathrm{A}$ and end of section $\mathrm{B}$ towards higher altitudes. More extreme peak values exceeding $18 \mathrm{ppbv}$ are found for the scenarios $v=100 \%, v=30 \%$ and $T+1 \mathrm{~K}$ compared to the MIPAS-STR observations.

Figures 10 and 11 show that mesoscale structures found in the MIPAS-STR results are reproduced by CLaMS to a high degree in general, while higher discrepancies are observed on the fine scale. Furthermore, the quality of the agreement 
(a)

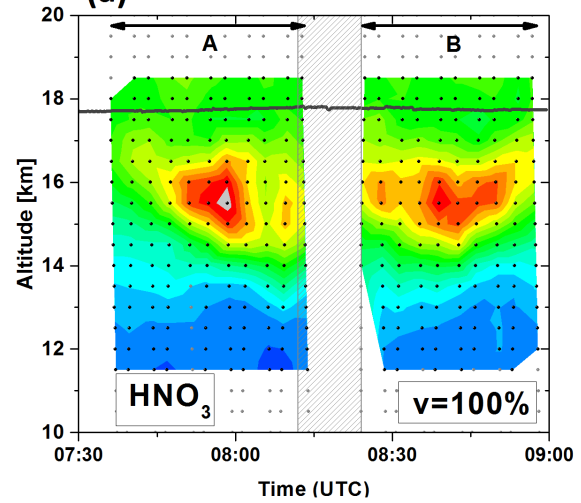

(c)

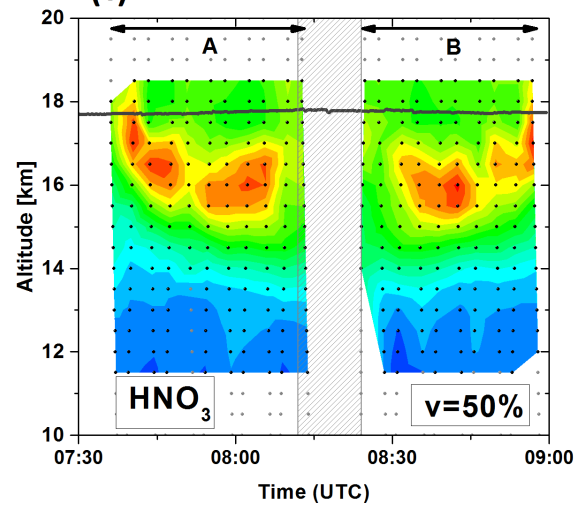

(e)

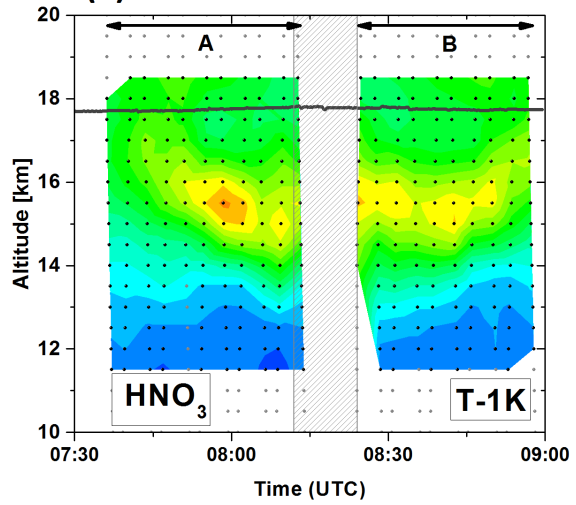

(g)

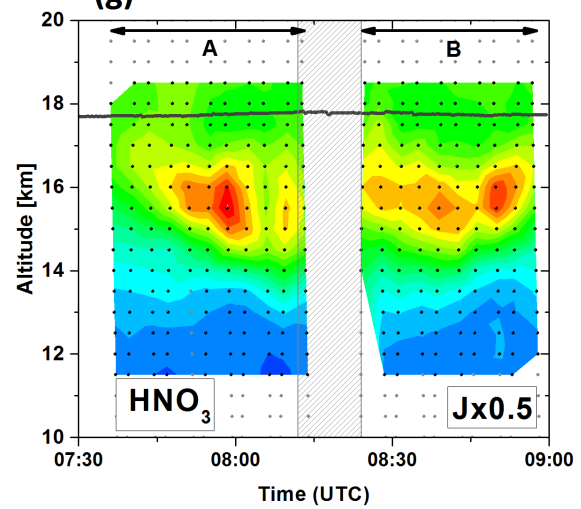

(b)

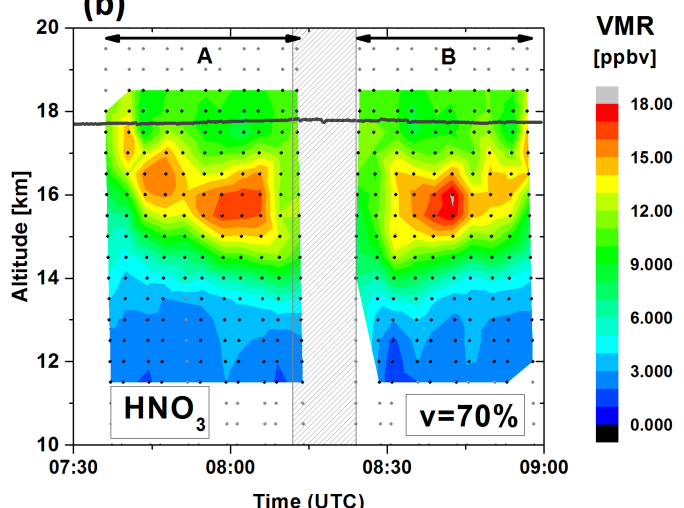

(d)

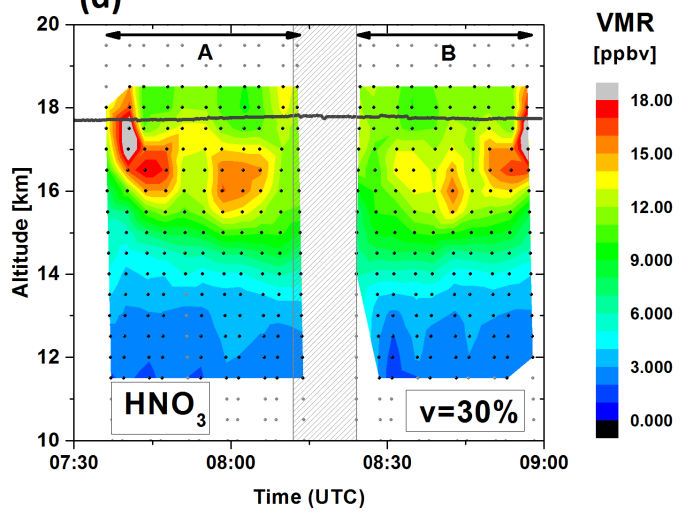

(f)

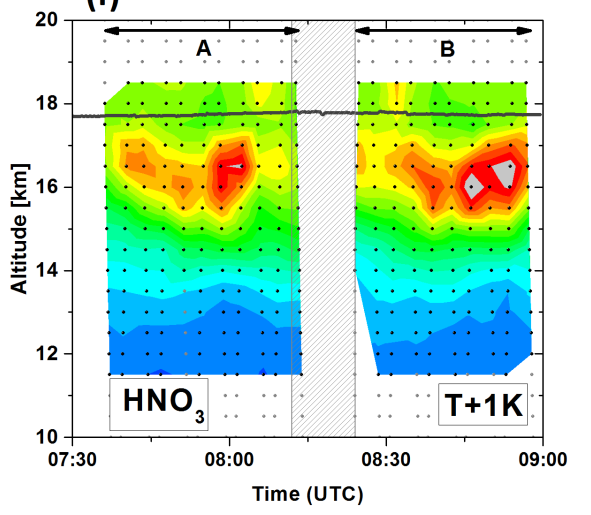

VMR

[ppbv]

18.00

15.00

12.00

9.000

6.000

3.000

0.000

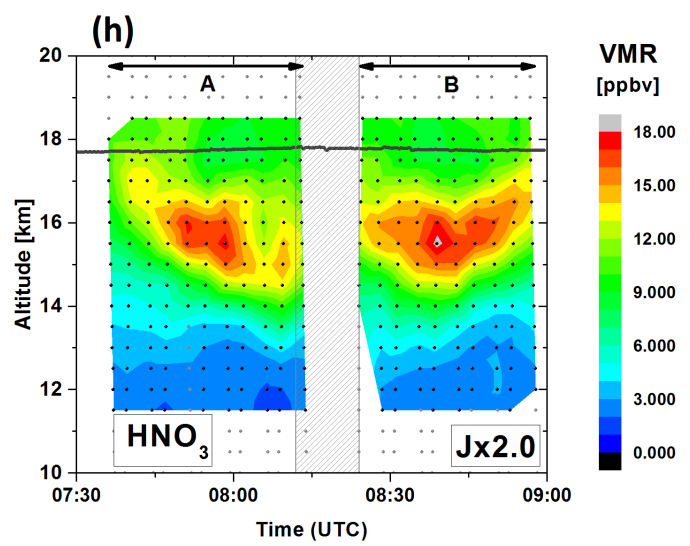

Figure 11. Same as Fig. 9 but for the flight on 30 January 2010 under conditions free of PSCs. 
between the MIPAS-STR observations and the CLaMS simulations varies for individual profiles and subsections.

\subsection{Ensemble profiles}

In Fig. 12a-h the ensemble profiles of gas-phase $\mathrm{HNO}_{3}$ corresponding to the flight on 25 January 2010 under PSC conditions are shown. The comparison is performed on levels of constant potential temperature to avoid biases from different pressure and temperature layering in the model domain compared to the atmosphere as seen by MIPAS-STR. All MIPASSTR data points and associated CLaMS data points for this flight are plotted where both retrieved temperature and $\mathrm{HNO}_{3}$ from MIPAS-STR are available. In the case of MIPAS-STR the potential temperature levels were calculated considering retrieved temperatures in combination with the corresponding pressure profiles extracted from ECMWF and used for the retrievals. Comparisons are shown again for the reference scenario, the scenarios considering the discussed reduced settling velocities, and the scenarios considering temperature biases and modified NAT nucleation rates.

Each plot shows (i) retrieved gas-phase $\mathrm{HNO}_{3}$ from MIPAS-STR, (ii) simulated gas-phase $\mathrm{HNO}_{3}$ from CLaMS associated with the geolocations of the MIPAS-STR data points and (iii) simulated passively transported $\mathrm{NO}_{\mathrm{y}}^{*}$ (without consideration of $\mathrm{HNO}_{3}$ condensation and vertical redistribution by NAT particle sedimentation) extracted from CLaMS for the same geolocations. The data points for $\mathrm{NO}_{\mathrm{y}}^{*}$ from CLaMS were smoothed horizontally and vertically in the same way as for $\mathrm{HNO}_{3}$. Simulated $\mathrm{NO}_{\mathrm{y}}^{*}$ can be compared directly to simulated and measured gas-phase $\mathrm{HNO}_{3}$ for the discussed flights, as $\mathrm{HNO}_{3}$ dominates the $\mathrm{NO}_{\mathrm{y}}$ budget in the lower stratosphere under Arctic winter conditions (e.g. Wiegele et al., 2009).

The comparison of measured and simulated gas-phase $\mathrm{HNO}_{3}$ relative to $\mathrm{NO}_{\mathrm{y}}^{*}$ shows, for all scenarios, reduced $\mathrm{HNO}_{3}$ mixing ratios at potential temperature levels higher than about $420 \mathrm{~K}$ (about $17.0 \mathrm{~km}$ altitude) and excess $\mathrm{HNO}_{3}$ at lower altitudes. Reduced $\mathrm{HNO}_{3}$ mixing ratios above the $420 \mathrm{~K}$ level in the model domain are the consequence of $\mathrm{HNO}_{3}$ being partially condensed in PSC particles and sedimentation of NAT particles, while the excess in $\mathrm{HNO}_{3}$ at lower levels results from evaporation of settled NAT particles. Accordingly, the simulation confirms that the MIPASSTR measurements associated with the flight on 25 January 2010 show vertical redistribution of $\mathrm{HNO}_{3}$ through denitrification and associated renitrification rather than dynamical features without these processes being involved.

While the overall scattering of the MIPAS-STR data points is mostly higher than for the simulation and the simulated renitrification maxima are located by trend at slightly lower potential temperature levels (typically by 10 to $20 \mathrm{~K}$ ), the amplitudes of the de- and renitrification structures show considerable agreement with the measurements for the scenarios $v=100 \%, v=70 \%$ and $v=50 \%$ (Fig. 12a-c). In contrast, the scenario $v=30 \%$ shows only a weak renitrification signal (Fig. 12d). A slightly more developed renitrification maximum compared to the other scenarios is found for the scenario $v=70 \%$, showing that this structure is not correlated linearly with the settling velocities. The scenario $T-1 \mathrm{~K}$ (Fig. 12e) and the scenarios with modified nucleation rates (Fig. 12g and h) show a comparable renitrification maximum to the reference scenario, while the scenario $T+1 \mathrm{~K}$ shows hardly any renitrification (Fig. 12f).

Figure 13a-h show the results of the comparison for the flight on 30 January 2010 under conditions free of PSCs and all previously condensed $\mathrm{HNO}_{3}$ being released back to the gas phase. Both the MIPAS-STR and CLaMS results show a strong maximum peaking around a potential temperature level of $400 \mathrm{~K}$. The comparison with simulated $\mathrm{NO}_{\mathrm{y}}^{*}$ allows for clear assignment to a strong renitrification structure, while the data points in the upper section above the $420 \mathrm{~K}$ level indicate effectively denitrified air. The observed maximum around $400 \mathrm{~K}$ exceeds simulated $\mathrm{NO}_{\mathrm{y}}^{*}$ by up to $7 \mathrm{ppbv}$. The CLaMS reference scenario $v=100 \%$ (Fig. 13a) reproduces the shape and the vertical extent of the observed maximum to a high degree. However, the simulated maximum appears slightly more narrow, and several data points exceed the maximum values inferred from the observations by up to 4 ppbv.

The scenario $v=70 \%$ (Fig. 13b) reproduces the shape of the observed ensemble profile significantly better. Except for some data points exceeding the observed distribution by up to about 2 to 3 ppbv above the $390 \mathrm{~K}$ level, all simulated values lie well within the scattering of the observations. The scenario $v=50 \%$ (Fig. 13c) also reproduces the observations reasonably. A few data points above the $390 \mathrm{~K}$ level, however, significantly exceed the simulated values by up to $4 \mathrm{ppbv}$, and the data points indicating effectively denitrified air (i.e. $\mathrm{HNO}_{3}$ mixing ratios lower than $\mathrm{NO}_{\mathrm{y}}^{*}$ ) above $420 \mathrm{~K}$ are reproduced to a lesser degree. The agreement of the $v=$ $30 \%$ (Fig. 13d) with the observations is significantly worse. The simulation shows lower $\mathrm{HNO}_{3}$ mixing ratios compared to the observations below $400 \mathrm{~K}$ and exceeds the observed values by up to $8 \mathrm{ppbv}$ at higher altitudes.

The scenarios considering temperature biases (Fig. 13ef) show significant differences from the reference scenario but no effective improvement. While the $T-1 \mathrm{~K}$ scenario reproduces the observations well above $420 \mathrm{~K}$, a more narrow and less developed maximum is found at lower altitudes. The scenario $T+1 \mathrm{~K}$ overestimates the observed maximum values around $410 \mathrm{~K}$ by up to $3 \mathrm{ppbv}$ and shows practically no effective denitrification above $420 \mathrm{~K}$.

The scenarios considering modified nucleation rates (Fig. 13g-h) show only small differences compared to the reference scenario. The overall shape of the observed ensemble profile is also fairly well reproduced by these scenarios, with the maximum values around $400 \mathrm{~K}$ being overestimated by up to about 3 ppbv. Furthermore, the scenario $\mathrm{J} \times 0.5$ reproduces the observed denitrification above $420 \mathrm{~K}$ 

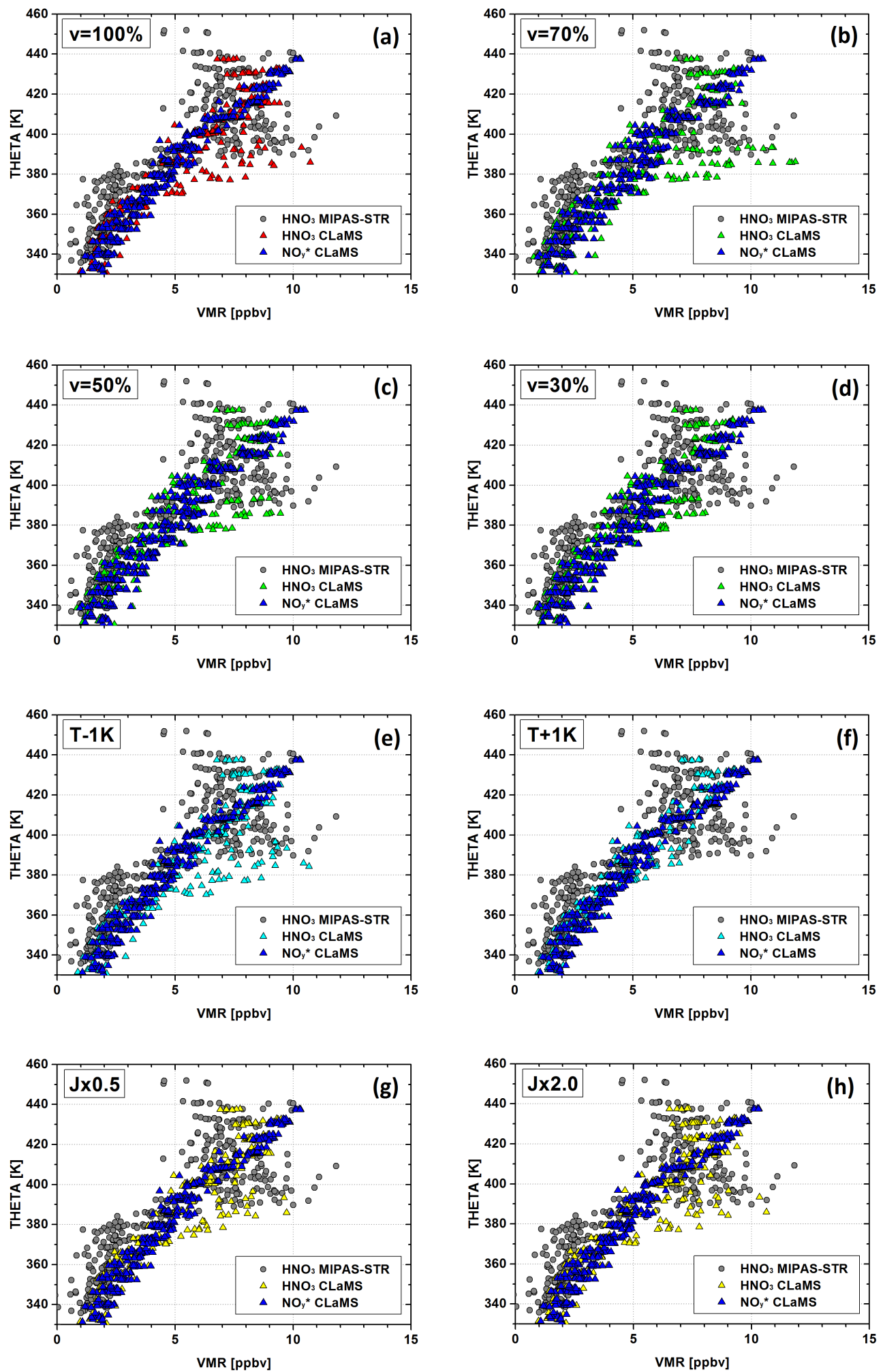

Figure 12. Comparison of the measured ensemble profile of gas-phase $\mathrm{HNO}_{3}$ from MIPAS-STR with the CLaMS reference simulation $(v=100 \%)$ and scenarios considering reduced settling velocities of NAT particles $(v=70 \%, v=50 \%$ and $v=30 \%)$, temperature biases of $\pm 1 \mathrm{~K}(T-1 \mathrm{~K}$ and $T+1 \mathrm{~K})$ and modified nucleation rates $(\mathrm{J} \times 0.5$ and $\mathrm{J} \times 2.0)$ for the PSC flight on 25 January 2010. $\mathrm{NO}_{\mathrm{y}}^{*}$ corresponds to simulated passive $\mathrm{NO}_{\mathrm{y}}$. 

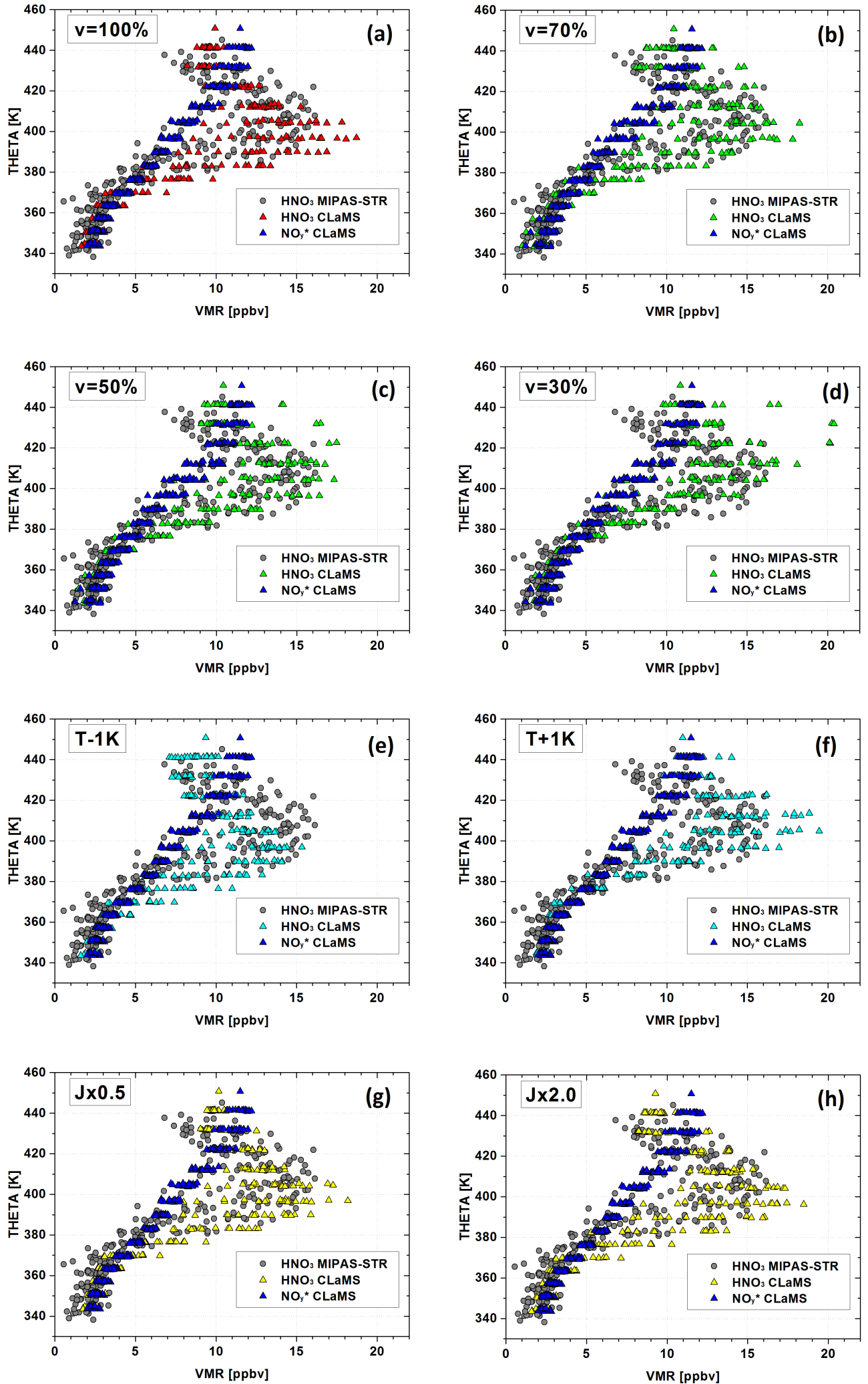

Figure 13. Same comparison as in Fig. 12 but for the flight on 30 January 2010 under conditions free of PSCs. 
to a slightly lesser degree compared to the reference simulation and the observations.

In summary, the simulations reproduce the major features (renitrification maxima below and denitrification minima above about $420 \mathrm{~K}$ ) of the observed ensemble profiles well in general (Figs. 12 and 13), while the quantitative agreement varies for the different scenarios. An important aspect is that the amplitudes of the changes resulting from the settling velocities reduced by a few tens of percent are of the same order or even higher compared to the effects from temperature biases of $\pm 1 \mathrm{~K}$ and the nucleation rates divided or multiplied by 2 .

For the flight on 30 January 2010, the best agreement is found for the scenario $v=70 \%$. Considerably worse agreement is found for the scenarios $v=30 \%$ and $T+1 \mathrm{~K}$ for both flights and the scenario $T-1 \mathrm{~K}$ for the flight on 30 January 2010. While the reference scenario $v=100 \%$ and the scenarios $\mathbf{J} \times 0.5$ and $\mathbf{J} \times 2.0$ show very similar results and reasonable agreement with the observations in general, these scenarios notably overestimate the maximum observed on 30 January 2010. Finally, the scenario $v=50 \%$ also shows reasonable agreement with the observations, with a slightly weaker renitrification maximum for the flight on 25 January 2010 and notable overestimation in the upper part of the profile for the flight on 30 January 2010.

\subsection{Discussion}

The presented results show that moderately reduced settling velocities of NAT particles between 100 and $50 \%$ relative to compact spherical particles were compatible with our observations of $\mathrm{HNO}_{3}$ redistribution. In the following we discuss which types of particles are expected to approximately show the settling characteristics considered in the different simulations in the context of the in situ observations (Fig. 1). We point out that conclusions from our study on the physical appearance of the particles are speculative due to the limitations of the comparison of the MIPAS-STR observations with the simulations and the fact that we can only derive indirect information on the particle characteristics via the parameter settling velocity. But we think that the discussion below gives a useful overview of the approximate relative settling velocities that have to be expected for some characteristic particle types in the considered size regime.

Approximately spherical particles with a net mass density lower than $1.62 \mathrm{~g} \mathrm{~cm}^{-3}$ (e.g. particles consisting of aggregates of smaller subunits) would have lower settling speeds compared to compact spherical particles of the same mass due to their larger hydrodynamic radii. Furthermore, compact (i.e. mass density of about $1.62 \mathrm{~g} \mathrm{~cm}^{-3}$ ) needle- or diskshaped particles would also have reduced settling velocities compared to mass-equivalent compact spherical particles (Westbrook, 2008). More complex combinations of particle shapes and mass densities are possible, but in the following we will focus on only these cases.
The first row of column 3 in Table 1 contains the mass density and dimensions of a typical compact spherical NAT particle capable of denitrification as simulated by CLaMS. The diameter of $10 \mu \mathrm{m}$ approximately corresponds to the sizes of the largest particles found in the CLaMS domain for the flight on 25 January 2010 under the conditions discussed in Sect. 3. Below, the sizes of potential spherical particles with reduced mass density having the same mass and approximately meeting the relative settling speed condition indicated in column 2 are listed.

It is pointed out that, in all modified CLaMS scenarios, the respective settling velocities of the simulated particles were multiplied by constant factors to approximate the settling speeds of alternative particle types. The Cunningham slip correction factors taken into account, however, were in all cases calculated corresponding to the equivalent compact spherical particles. When calculating the sizes of the massequivalent alternative particle types approximately meeting the relative settling velocity conditions indicated in column 2 , only the mass equivalence and the Stokes equation were considered. Contributions from different Cunningham corrections applying to larger diameters or different sizes as a consequence of reduced particle mass density or alternative particle shapes were not considered for conversion. However, the slip correction factor for a hypothetic low mass density spherical particle with a diameter of $20 \mu \mathrm{m}$ is only by about $5 \%$ lower compared to that of a compact spherical particle with a diameter of $10 \mu \mathrm{m}$. Furthermore, when transferring the mass of a compact spherical particle of similar size into a compact moderately aspheric particle (see below), the effective difference in the Cunningham correction is expected to be of the same order. As the aim of this work is only to give typical sizes of certain particle classes approximately fulfilling the indicated relative settling velocity conditions rather than a full quantitative assessment, these simplifications are acceptable here.

According to Table 1, a spherical NAT particle with a diameter of $14 \mu \mathrm{m}$ and a mass density of $0.56 \mathrm{~g} \mathrm{~cm}^{-3}$ would have a settling velocity of approximately $70 \%$ compared to a mass-equivalent compact spherical NAT particle in the reference scenario (density of $1.62 \mathrm{~g} \mathrm{~cm}^{-3}$ and diameter of $10 \mu \mathrm{m})$. Similarly, mass-equivalent spherical particles with diameters of 20 and $33 \mu \mathrm{m}$ and the indicated mass densities would have relative settling velocities of approximately 50 and $30 \%$.

Columns 4 and 5 in Table 1 give the dimensions of the potential of columnar needle- and disk-shaped particles containing the same mass as the indicated spherical particles and approximately meeting the indicated relative settling velocity conditions. The aspect ratio here is the ratio between height and diameter of a hypothetical cylinder (i.e. column or disk). For estimating the sizes of the indicated aspheric particle types associated with the discussed relative settling velocity conditions the respective hydrodynamic radii were approximated by the corresponding capacitances according 
to Westbrook (2008). The relation between particle size and capacitance for cylindrical particles was taken from Smythe (1962). Furthermore, for the discussed hypothetical diskshaped particles, the approximation for horizontally oriented disks as discussed by Westbrook (2008, references therein) was taken into account, since potential disk-shaped NAT particles in the considered size regime might show preferentially horizontal orientations under stratospheric conditions.

According to Table 1, a compact columnar (i.e. needleshaped) particle with a length of $34 \mu \mathrm{m}$ and an aspect ratio of 7.6 would have a settling velocity of approximately $70 \%$ compared to a mass-equivalent spherical particle in the reference simulation. Relative settling velocities of 50 and $30 \%$ would approximately apply to columnar particles that have lengths of 68 and $168 \mu \mathrm{m}$ and that are characterised by the indicated aspect ratios. Furthermore, compact mass-equivalent disk-shaped particles with diameters of 11,21 and $38 \mu \mathrm{m}$ and the indicated aspect ratios would have relative settling velocities of about 70,50 and $30 \%$. We mention, however, that the simplification regarding the Cunningham slip correction has to be regarded critically for the extreme cases.

For the flight on 25 January 2010, maximum particle masses equivalent to that of compact spherical particles with diameters not larger than 11 to $12 \mu \mathrm{m}$ are expected as a consequence of limited growing time (compare Sect. 3). From the in situ observations shown in Fig. 1, maximum particle sizes around $30 \mu \mathrm{m}$ were obtained for this flight. According to Table 1, (i) spherical particles with a rather low mass density of about $0.04 \mathrm{~g} \mathrm{~cm}^{-3}$ and a relative settling velocity of about $30 \%$, (ii) compact columnar particles with an aspect ratio of about 7.6 and a relative settling velocity of $70 \%$, and (iii) compact disk-shaped particles with an aspect ratio of 0.01 and a relative settling velocity of $30 \%$ would have maximum dimensions (diameter of a sphere or circular disk or height of a circular cylinder, respectively) similar to the maximum sizes indicated by the in situ particle observations. The considered spherical and disk-shaped particles that were characterised by relative settling velocities of about $50 \%$ also would have maximum dimensions significantly larger than the corresponding particles in the reference simulation, but were still smaller compared to the most extreme sizes indicated by the in situ observations.

The comparisons between simulated and measured overall $\mathrm{HNO}_{3}$ redistribution show reasonable agreement for simulations considering relative settling velocities between 100 and $50 \%$ (best agreement by trend for the $70 \%$ scenario). In contrast, the simulation considering relative settling velocities of $30 \%$ significantly underestimates the observed vertical redistribution of $\mathrm{HNO}_{3}$.

We point out that it is not clear how (i) the maximum sizes inferred from the FSSP-100 measurements have to be interpreted in the context of significantly aspheric particles, and (ii) limitations of the comparison between the MIPAS-STR measurements and the CLaMS simulations affect the discussed results. However, combining the presented results into one picture, we speculate that the large particles indicated by the in situ observations during the flight on 25 January 2010 were compact, aspheric significantly elongated NAT particles. Such particles could grow to larger maximum extensions in a shorter time compared to compact spherical particles having the same mass, and, at the same time, such particles were expected to have moderately reduced relative settling velocities compared to mass-equivalent compact spherical particles.

\section{Conclusions}

This study analyses the combination of airborne in situ particle observations, airborne remote sensing observations of gas-phase $\mathrm{HNO}_{3}$, temperature and PSC coverage as well as Lagrangian simulations of vertical $\mathrm{HNO}_{3}$ redistribution by NAT particles associated with the Arctic winter stratosphere at the end of January 2010. The observations and simulations suggest that an ongoing denitrification process with NAT particles involved was observed during the Geophysica PSC flight on 25 January 2010. The analysis of the formation conditions of large particles detected by the FSSP-100 utilising particle backward trajectory analyses shows that the observations are not consistent with compact spherical NAT particles. Ice particles and NAT-coated ice particles or icecoated NAT particles appear unlikely due to too warm temperatures, as indicated consistently by the observations and the model. We investigated the hypothesis that the simulation reproduces the NAT particle masses in a realistic way, but that real NAT particles have larger apparent sizes compared to compact spherical particles, e.g. due to non-compact morphology or aspheric shape. We focused on the fact that reduced settling velocities have to expected for such particles when compared to compact spherical particles, altering the vertical redistribution of $\mathrm{HNO}_{3}$.

Using simulations by CLaMS and observations of MIPASSTR, we studied the impact of reduced settling velocities of large NAT particles on vertical $\mathrm{HNO}_{3}$ redistribution. The reference scenario and the scenarios considering reduced relative settling velocities of 70 and $50 \%$ result in reasonable agreement with the observed overall $\mathrm{HNO}_{3}$ redistribution. While the best agreement is found for the $70 \%$ scenario, relative settling velocities of $30 \%$ result in a significant underestimation of the vertical $\mathrm{HNO}_{3}$ redistribution by the simulation. Sensitivity simulations considering temperature biases of $\pm 1 \mathrm{~K}$ show no effective improvement in the agreement between simulation and observation, while simulations with the NAT nucleation rates multiplied by factors of 0.5 and 2.0 change the results of the simulations only marginally.

We find that changing the settling velocities of the simulated NAT particles by a few tens of percent has comparable effects on $\mathrm{HNO}_{3}$ redistribution in terms of amplitudes of $\mathrm{HNO}_{3}$ maxima and minima when compared to the discussed scenarios considering temperature biases and 
modified nucleation rates. Accordingly, the choice of the simulated settling velocities of NAT particles significantly affects the resulting vertical $\mathrm{HNO}_{3}$ redistribution.

One possible interpretation of the large particle sizes observed during the flight on 25 January 2010 in the context of this study would be that these particles were compact and considerably aspheric. Such particles would have larger maximum extensions than compact spherical particles of the same masses and were expected to have moderately reduced settling velocities at the same time. However, we point out that no quantitative conclusions on the physical appearance of the particles can be drawn from our study and that the effect of considerably aspheric particles on the interpretation of the in situ observations is unclear. Further observations and advanced evaluation techniques are necessary to determine the properties of the particles involved in denitrification unambiguously.

However, our results show that an accurate knowledge of the settling velocities of NAT particles involved in denitrification is important for quantitative simulations of vertical $\mathrm{HNO}_{3}$ redistribution.

Acknowledgements. RECONCILE was funded by the European Commission under the grant number RECONCILE-226365-FP7ENV-2008-1. We thank the RECONCILE coordination team, the Myasishchev Design Bureau and Enviscope for making the RECONCILE field campaign a success. ECMWF data for the MIPAS-STR retrievals were obtained via NILU's NADIR database. The CLaMS simulations were performed using computing time granted on the supercomputer JUROPA at Jülich Supercomputing Centre (JSC) under the VSR project ID JICG11. We acknowledge support by the Deutsche Forschungsgemeinschaft and Open Access Publishing Fund of the Karlsruhe Institute of Technology.

The service charges for this open access publication have been covered by a Research Centre of the Helmholtz Association.

Edited by: F. Khosrawi

\section{References}

Borrmann, S., Luo, B., and Mishchenko, M.: The application of the T-matrix method to the measurement of aspherical particles with forward scattering optical particle counters, J. Aerosol Sci., 31, 789-799, 2000.

Carslaw, K. S., Kettleborough, J. A., Northway, M. J., Davies, S., Gao, R., Fahey, D. W., Baumgardner, D. G., Chipperfield, M. P., and Kleinböhl, A.: A vortex-scale simulation of the growth and sedimentation of large nitric acid hydrate particles, J. Geophys. Res., 107, 8300, doi:10.1029/2001JD000467, 2002.

de Reus, M., Borrmann, S., Bansemer, A., Heymsfield, A. J., Weigel, R., Schiller, C., Mitev, V., Frey, W., Kunkel, D., Kürten, A., Curtius, J., Sitnikov, N. M., Ulanovsky, A., and Ravegnani, F.: Evidence for ice particles in the tropical strato- sphere from in-situ measurements, Atmos. Chem. Phys., 9, 6775-6792, doi:10.5194/acp-9-6775-2009, 2009.

Dörnbrack, A., Pitts, M. C., Poole, L. R., Orsolini, Y. J., Nishii, K., and Nakamura, H.: The 2009-2010 Arctic stratospheric winter general evolution, mountain waves and predictability of an operational weather forecast model, Atmos. Chem. Phys., 12, 36593675, doi:10.5194/acp-12-3659-2012, 2012.

Drdla, K., Turco, R. P., and Elliott, S.: Heterogeneous chemistry on Antarctic polar stratospheric clouds: a microphysical estimate of the extent of chemical processing, J. Geophys. Res., 98, 89658981, doi:10.1029/93JD00164, 1993.

Fahey, D. W., Gao, R. S., Carslaw, K. S., Kettleborough, J., Popp, P. J., Northway, M. J., Holecek, J. C., Ciciora, S. C., McLaughlin, R. J., Thompson, T. L., Winkler, R. H., Baumgardner, D. G., Gandrud, B., Wennberg, P. O., Dhaniyala, S., McKinney, K., Peter, T., Salawitch, R. J., Bui, T. P., Elkins, J. W., Webster, C. R., Atlas, E. L., Jost, H., Wilson, J. C., Herman, R. L., Kleinbohl, A., and von Konig, M.: The detection of large $\mathrm{HNO}_{3}-$ containing particles in the winter arctic stratosphere, Science, 291, 1026-1031, 2001.

Grooß, J.-U., Günther, G., Müller, R., Konopka, P., Bausch, S., Schlager, H., Voigt, C., Volk, C.M., and Toon, G. C.: Simulation of denitrification and ozone loss for the Arctic winter 2002/2003, Atmos. Chem. Phys., 5, 1437-1448, doi:10.5194/acp-5-14372005, 2005.

Grooß, J.-U., Engel, I., Borrmann, S., Frey, W., Günther, G., Hoyle, C. R., Kivi, R., Luo, B. P., Molleker, S., Peter, T., Pitts, M. C., Schlager, H., Stiller, G., Vömel, H., Walker, K. A., and Müller, R.: Nitric acid trihydrate nucleation and denitrification in the Arctic stratosphere, Atmos. Chem. Phys., 14, 1055-1073, doi:10.5194/acp-14-1055-2014, 2014.

Grothe, H., Tizek, H., Waller, D., and Stokes, D. J.: The crystallization kinetics and morphology of nitric acid trihydrate, Phys. Chem. Chem. Phys., 8, 2232-2239, doi:10.1039/B601514J, 2006.

Hanson, D. and Mauersberger, K.: Laboratory studies of the nitric acid trihydrate: implications for the south polar stratosphere, Geophys. Res. Lett., 15, 855-858, doi:10.1029/GL015i008p00855, 1988.

Höpfner, M.: Study on the impact of polar stratospheric clouds on high resolution mid-IR limb emission spectra, J. Quant. Spectrosc. Radiat. Transfer, 83, 93-107, 2004.

Höpfner, M., Blom, C. E., Echle, G., Glatthor, N., Hase, F., and Stiller, G.: Retrieval simulations for MIPAS-STR measurements, edited by: Smith, W. L., IRS 2000: Current Problems in Atmospheric Radiation; Proc. of the Internat. Radiation Symp., St. Petersburg, Russia, 24-29 July 2000 Hampton, Va.: DEEPAK Publ., 2001.

Hoyle, C. R., Engel, I., Luo, B. P., Pitts, M. C., Poole, L. R., Grooß, J.-U., and Peter, T.: Heterogeneous formation of polar stratospheric clouds - Part 1: Nucleation of nitric acid trihydrate (NAT), Atmos. Chem. Phys., 13, 9577-9595, doi:10.5194/acp13-9577-2013, 2013.

Libbrecht, K. G.: The physics of snow crystals, Rep. Prog. Phys., 68, 855-895, doi:10.1088/0034-4885/68/4/R03, 2005.

Keyser, L. F. and Leu, M.-T.: Morphology of Nitric Acid and Water Ice Films, Microsc. Res. Tech., 25, 434-438, doi:10.1002/jemt.1070250514, 1993. 
Khaykin, S. M., Engel, I., Vömel, H., Formanyuk, I. M., Kivi, R., Korshunov, L. I., Krämer, M., Lykov, A. D., Meier, S., Naebert, T., Pitts, M. C., Santee, M. L., Spelten, N., Wienhold, F. G., Yushkov, V. A., and Peter, T.: Arctic stratospheric dehydration - Part 1: Unprecedented observation of vertical redistribution of water, Atmos. Chem. Phys., 13, 11503-11517, doi:10.5194/acp13-11503-2013, 2013.

Khosrawi, F., Urban, J., Pitts, M. C., Voelger, P., Achtert, P., Kaphlanov, M., Santee, M. L., Manney, G. L., Murtagh, D., and Fricke, K.-H.: Denitrification and polar stratospheric cloud formation during the Arctic winter 2009/2010, Atmos. Chem. Phys., 11, 8471-8487, doi:10.5194/acp-11-8471-2011, 2011.

Marti, J. J. and Mauersberger, K.: Evidence for nitric acid pentahydrate formed under stratospheric conditions, J. Phys. Chem., 98, 6897-6899, doi:10.1021/j100079a001, 1994.

Molleker, S., Borrmann, S., Schlager, H., Luo, B., Frey, W., Klingebiel, M., Weigel, R., Ebert, M., Mitev, V., Matthey, R., Woiwode, W., Oelhaf, H., Dörnbrack, A., Stratmann, G., Grooß, J.-U., Günther, G., Vogel, B., Müller, R., Krämer, M., Meyer, J., and Cairo, F.: Microphysical properties of synoptic-scale polar stratospheric clouds: in situ measurements of unexpectedly large $\mathrm{HNO}_{3}$ containing particles in the Arctic vortex, Atmos. Chem. Phys., 14, 10785-10801, doi:10.5194/acp-14-10785-2014, 2014.

Müller, R. and Peter, Th.: The numerical modelling of the sedimentation of polar stratospheric cloud particles, Ber. Bunsen. Phys. Chem., 96, 353-361, 1992.

Nash, E. R., Newman, P. A., Rosenfield, J. E., and Schoeberl, M. R.: An objective determination of the polar vortex using Ertel's potential vorticity, J. Geophys. Res., 101, 9471-9478, 1996.

Peter, T. and Grooß, J.-U.: Polar Stratospheric Clouds and Sulfate Aerosol Particles: Microphysics, Denitrification and Heterogeneous Chemistry, in: Stratospheric Ozone Depletion and Climate Change, edited by: Müller, R., RSC Publishing, UK, 108-144, 2012

Phillips, C.: A technique for the numerical solution of certain integral equations of the first kind, J. Assoc. Comput. Math., 9, 84-97, 1962.

Piesch, C., Gulde, T., Sartorius, C., Friedl-Vallon, F., Seefeldner, M., Wölfel, M., Blom, C. E., and Fischer, H.: Design of a MIPAS Instrument for High-Altitude Aircraft, Proc. of the 2nd Internat. Airborne Remote Sensing Conference and Exhibition, ERIM, Ann Arbor, MI, Vol. II, 199-208, 24-27 June 1996, San Francisco, 1996.

Pitts, M. C., Poole, L. R., Dörnbrack, A., and Thomason, L. W.: The 2009-2010 Arctic polar stratospheric cloud season: a CALIPSO perspective, Atmos. Chem. Phys., 11, 2161-2177, doi:10.5194/acp-11-2161-2011, 2011.

Pruppacher, H. R. and Klett, J. D.: Microphysics of Clouds and Precipitation, 2nd Edn., Kluwer Academic Publishers, Dordrecht, 1997.

Purser, R. J. and Huang, H.-L.: Estimating effective data density in a satellite retrieval or an objective analysis, J. App. Meteorol., 32, 1092-1107, 1993.

Rodgers, C. D.: Inverse Methods for Atmospheric Sounding: theory and Practice, vol. 2 of Series on Atmospheric, Oceanic and Planetary Physics, edited by: Taylor, F. W., World Scientific, Singapore, 2000.

Smythe, W. R.: Charged right circular cylinder, J. Appl. Phys., 33, 2966-2967, doi:10.1063/1.1722514, 1962.
Solomon, S.: Stratospheric ozone depletion: a review of concepts and history, Rev. Geophys., 37, 275-316, doi:10.1029/1999RG900008, 1999.

Spang, R., Remedios, J. J., and Barkley, M. P.: Colour indices for the detection and differentiation of cloud types in infra-red limb emission spectra, Adv. Space Res., 33, 1041-1047, 2004.

Stiller, G. P., von Clarmann, T., Funke, B., Glatthor, N., Hase, F., Höpfner, M., and Linden, A.: Sensitivity of trace gas abundances retrievals from infrared limb emission spectra to simplifying approximations in radiative transfer modelling, J. Quant. Spectrosc. Ra., 72, 249-280, doi:10.1016/S0022-4073(01)00123-6, 2002.

Tabazadeh, A. and Toon, O. B.: The presence of metastable $\mathrm{HNO}_{3} / \mathrm{H}_{2} \mathrm{O}$ solid phases in the stratosphere inferred from ER2 data, J. Geophys. Res., 101, 9071-9078, doi:10.1029/96JD00062, 1996.

Tikhonov, A.: On the Solution of Incorrectly Stated Problems and a Method of Regularisation, Dokl. Acad. Nauk SSSR, 151, 501504, 1963.

Ungermann, J., Kalicinsky, C., Olschewski, F., Knieling, P., Hoffmann, L., Blank, J., Woiwode, W., Oelhaf, H., Hösen, E., Volk, C. M., Ulanovsky, A., Ravegnani, F., Weigel, K., Stroh, F., and Riese, M.: CRISTA-NF measurements with unprecedented vertical resolution during the RECONCILE aircraft campaign, Atmos. Meas. Tech., 5, 1173-1191, doi:10.5194/amt-5-11732012, 2012.

von Hobe, M., Bekki, S., Borrmann, S., Cairo, F., D’Amato, F., Di Donfrancesco, G., Dörnbrack, A., Ebersoldt, A., Ebert, M., Emde, C., Engel, I., Ern, M., Frey, W., Genco, S., Griessbach, S., Grooß, J.-U., Gulde, T., Günther, G., Hösen, E., Hoffmann, L., Homonnai, V., Hoyle, C. R., Isaksen, I. S. A., Jackson, D. R., Jánosi, I. M., Jones, R. L., Kandler, K., Kalicinsky, C., Keil, A., Khaykin, S. M., Khosrawi, F., Kivi, R., Kuttippurath, J., Laube, J. C., Lefèvre, F., Lehmann, R., Ludmann, S., Luo, B. P., Marchand, M., Meyer, J., Mitev, V., Molleker, S., Müller, R., Oelhaf, H., Olschewski, F., Orsolini, Y., Peter, T., Pfeilsticker, K., Piesch, C., Pitts, M. C., Poole, L. R., Pope, F. D., Ravegnani, F., Rex, M., Riese, M., Röckmann, T., Rognerud, B., Roiger, A., Rolf, C., Santee, M. L., Scheibe, M., Schiller, C., Schlager, H., Siciliani de Cumis, M., Sitnikov, N., Søvde, O. A., Spang, R., Spelten, N., Stordal, F., Sumińska-Ebersoldt, O., Ulanovski, A., Ungermann, J., Viciani, S., Volk, C. M., vom Scheidt, M., von der Gathen, P., Walker, K., Wegner, T., Weigel, R., Weinbruch, S., Wetzel, G., Wienhold, F. G., Wohltmann, I., Woiwode, W., Young, I. A. K., Yushkov, V., Zobrist, B., and Stroh, F.: Reconciliation of essential process parameters for an enhanced predictability of Arctic stratospheric ozone loss and its climate interactions (RECONCILE): activities and results, Atmos. Chem. Phys., 13, 9233-9268, doi:10.5194/acp-13-9233-2013, 2013.

Wagner, R., Möhler, O., Saathoff, H., Stetzer, O., and Schurath, U.: Infrared spectrum of nitric acid dihydrate: influence of particle shape, J. Phys. Chem. A, 109, 2572-2581, doi:10.1021/jp044997u, 2005.

Westbrook, C. D.: The fall speeds of sub- $100 \mu \mathrm{m}$ ice crystals, Q. J. Roy. Meteor. Soc., 134, 1243-1251, 2008.

Wiegele, A., Kleinert, A., Oelhaf, H., Ruhnke, R., Wetzel, G., Friedl-Vallon, F., Lengel, A., Maucher, G., Nordmeyer, H., and Fischer, H.: Spatio-temporal variations of $\mathrm{NO}_{\mathrm{y}}$ species in the northern latitudes stratosphere measured with the balloon- 
borne MIPAS instrument, Atmos. Chem. Phys., 9, 1151-1163, doi:10.5194/acp-9-1151-2009, 2009.

Woiwode, W., Oelhaf, H., Gulde, T., Piesch, C., Maucher, G., Ebersoldt, A., Keim, C., Höpfner, M., Khaykin, S., Ravegnani, F., Ulanovsky, A. E., Volk, C. M., Hösen, E., Dörnbrack, A., Ungermann, J., Kalicinsky, C., and Orphal, J.: MIPAS-STR measurements in the Arctic UTLS in winter/spring 2010: instrument characterization, retrieval and validation, Atmos. Meas. Tech., 5, 1205-1228, doi:10.5194/amt-5-1205-2012, 2012.
Woiwode, W.: Qualification of the airborne FTIR spectrometer MIPAS-STR and study on denitrification and chlorine deactivation in Arctic winter 2009/10, PhD Thesis, Karlsruhe Institute of Technology, Faculty of Chemistry and Biosciences, Karlsruhe, Germany, 2013.

Worsnop, D. R., Zahniser, M. S., Fox, L. E., and Wofsy, S. C.: Vapor Pressures of Solid Hydrates of Nitric Acid: implications for Polar Stratospheric Clouds, Science, 259, 71-74, 1993. 